\title{
The phase diagram of a frustrated Heisenberg model: from disorder to order and back again
}

\author{
Michel M. J. Miranda, ${ }^{1,2,3}$ Igor C. Almeida, ${ }^{1}$ Eric C. Andrade, ${ }^{1}$ and José A. Hoyos ${ }^{1}$ \\ ${ }^{1}$ Instituto de Física de São Carlos, Universidade de São Paulo, C.P. 369, 13560-970, São Carlos, SP, Brazil \\ ${ }^{2}$ Institut für Theoretische Physik and Würzburg-Dresden Cluster of Excellence ct.qmat, \\ Technische Universität Dresden, 01062 Dresden, Germany \\ ${ }^{3}$ Max-Planck-Institut für Chemische Physik fester Stoffe, Nöthnitzer Straße 40, 01187 Dresden, Germany
}

(Dated: August 4, 2021)

\begin{abstract}
We study the effects of bond and site disorder in the classical $J_{1}-J_{2}$ Heisenberg model on a square lattice in the order-by-disorder frustrated regime $2 J_{2}>\left|J_{1}\right|$. Combining symmetry arguments, numerical energy minimization and large scale Monte Carlo simulations, we establish that the finite-temperature Ising-type transition of the clean system is destroyed in the presence of any finite concentration of impurities. We explain this finding via a random-field mechanism which generically emerges in systems where disorder locally breaks the same real-space symmetry spontaneously globally broken by the associated order parameter. We also determine that the phase replacing the clean one is a paramagnet polarized in the nematic glass order with non-trivial magnetic response. This is because disorder also induces non-collinear spin-vortex-crystal order and produces a conjugated transverse dipolar random field. As a result of these many competing effects, the associated magnetic susceptibilities are non-monotonic functions of the temperature. As a further application of our methods, we show the generation of random axes in other frustrated magnets with broken $\mathrm{SU}(2)$ symmetry. We also discuss the generality of our findings and their relevance to experiments.
\end{abstract}

Published in Phys. Rev. B 104, 054201 (2021); DOI: 10.1103/PhysRevB.104.054201

\section{INTRODUCTION}

Understanding the effects of quenched disorder is a long-standing and fundamental problem in condensed matter systems. It has long been recognized that it can qualitatively modify the properties of the phases near a phase transition $[1,2]$, change the critical behavior of continuous phase transitions [3], turn a first-order phase transition into a continuous one [4], and even destroy the long-range order of the phase itself [5], among other effects (for a review, see, e.g., Ref. 6).

All these effects can be understood in the context of single-order-parameter field theory in which disorder adds a random component to either the conjugate field or to the mass terms. In non-frustrated magnetic systems (such as those described by the Ising or Heisenberg models), the random mass term is generated by any inhomogeneities preserving the symmetry of the Hamiltonian interactions such as site dilution or bond defects. This term is particularly important near phase transitions yielding to the so-called Griffiths singularities $[1,2]$ in addition possibly changing the universality class of a continuous phase transition [3]. (For more recent developments, see, e.g., Ref. 7 and references therein.)

The effects of random conjugate fields can be even more dramatic. At sufficiently low dimensions, they produce non-perturbative effects completely destroying the phase transition [5]. However, random fields are rarely realized in non-frustrated systems because site and bond disorder generally preserve the symmetry broken by the resulting magnetic order. In frustrated systems, conversely, the resulting order commonly breaks a real-space lattice symmetry which is usually locally broken by the impurities. Therefore, a direct coupling between disorder and the order parameter field is expected. How this coupling manifests itself in the associated field theory depends on how the order-parameter degeneracy is lifted by disorder. For a simple $\mathrm{Z}_{2}$-symmetric field, only a random field can be generated. For a $\left(\mathrm{Z}_{n} \otimes \mathrm{Z}_{2}\right)$-symmetric field with disorder lifting the associated degeneracy down to a $\mathrm{Z}_{2}$ one, the generated term is then a random easy axis.

In the frustrated $J_{1}-J_{2}$ Ising model, bond and site disorder generate a random field term precluding any longrange stripe order in dimensions $d \leq 2$ [8]. Later, it was shown that part of this result also applies to the $J_{1}-J_{2}$ Heisenberg model: bond disorder generates a random nematic field term [9]. However, it became unclear whether site vacancies produce the same outcome because (i) the random-field term is not generated in the one-loop approximation [9] and (ii) there is another effect taking place: due to the continuous symmetry character of the interactions, site vacancies nucleate a non-collinear spin-vortex-crystal order via the order-by-disorder mechanism [10]. In addition, Monte Carlo numerical simulations were interpreted as supporting nematic long-range order in the regime of low dilution [11]. ${ }^{1}$

Clearly, from the symmetry perspective, site and bond disorder should produce qualitatively the same effects in this scenario. Thus, the aforementioned reason (i) is re-

\footnotetext{
1 The nematic and spin-vortex-crystal orders are commonly designated by collinear (or columnar antiferromagnetic) and anticollinear order, respectively.
} 
futed. A random-field term is expected in higher orders of approximation. Reason (ii) is less clear, but the symmetry argument can be played in the other way around: the spin-vortex-crystal order is also nucleated by bond disorder, an effect not explored in the literature so far.

Finally, we point out to a recent development which makes this problem even less clear. It was shown that more subtle situations can also generate a coupling between disorder and the order-parameter field. When the ordered state breaks inversion symmetry (such as the spin-vortex-crystal one), a single-bond defect (which does not break this symmetry) generates a slowly decaying transverse dipolar field [12]. In the presence of a finite concentration of bond defects, the non-collinear ordered state is destroyed by these random dipolar fields.

Having enumerated all these uncertainties, a deeper understanding of the effects of disorder in frustrated magnets is desirable. In this work, we revisit the effects of site and bond disorder on the $J_{1}-J_{2}$ classical Heisenberg model and show that any finite amount of disorder (either site or bond impurities) precludes the nematicparamagnet phase transition due to generation of random fields. Although the effect of a single-site vacancy is different from that of a single-bond defect, the effects of any finite fraction of these impurities are equivalent because it is possible to find configurations of these impurities breaking the same real-space symmetries. In addition, we show that the resulting paramagnet is polarized in the nematic spin-cluster glass order as the system is broken into domains exhibiting local nematic order. ${ }^{2}$ The domain walls exhibit non-collinear competing order with temperature-dependent thickness, yielding a nontrivial behavior to the susceptibilities. At zero temperature, the spin configuration remains coplanar for weak disorder. The spin-vortex-crystal order is destroyed by the transverse dipolar random fields resulting in a spinvortex-crystal glass. However, the associated vestigial spin-vorticity density-wave order is perturbatively stable against these fields. Our conclusions are based on a simple symmetry analysis for determining how disorder locally lifts the degeneracy of the order-parameter manifold and are confirmed by numerical energy minimization and Monte Carlo simulations.

The remainder of this paper is organized as follows. In Sec. II we introduce the model and review the order-bydisorder mechanism which is responsible for stabilizing the long-range nematic (collinear) order. In Sec. III we study the effects of bond and site disorder. We review the known effects of single impurities and establish the equivalence of their effects when a finite density of them is present. We also show that random dipolar fields conjugate to the spin-vortex-crystal order is generated. Fi-

\footnotetext{
${ }^{2}$ We adopt the terminology of Ref. 13 in which the spin-cluster glass differs from the usual Edwards-Anderson spin glass (or other glasses) as the magnetic correlation length (the nematic domain) in the former can be arbitrarily large.
}

nally, we determine the phase diagram and characterize the resulting thermal state. In Sec. IV we present extensive numerical simulations to support our scenario. As an example of our method, in the Appendix, we analyze a class of XY frustrated magnets with off-diagonal (spinorbit-induced) couplings and show the disorder-induced generation of a random easy-axis term. In $d=2$, this term destroys long-range order and in $d=3$, it stabilizes a cluster-spin-glass phase for sufficiently strong disorder. Finally, in Sec. V we present our concluding remarks and briefly discuss the implication of our results to the case of quantum spins and materials compounds.

\section{THE MODEL AND THE ENTROPIC SELECTION}

We revisit the random $J_{1}-J_{2}$ classical Heisenberg model,

$$
\mathcal{H}=\sum_{\langle i, j\rangle} J_{1, i j} \mathbf{S}_{i} \cdot \mathbf{S}_{j}+\sum_{\langle\langle i, j\rangle\rangle} J_{2, i j} \mathbf{S}_{i} \cdot \mathbf{S}_{j},
$$

on a square lattice of $N=L \times L$ sites with periodic boundary conditions. The classical spins $\mathbf{S}_{i}$ are threecomponent unity vectors. The exchange interactions $J_{1(2), i j}$ are between nearest- (next-nearest-) $(i, j)$ neighbor sites quantified by $J_{\alpha, i j}=J_{\alpha}+\delta J_{\alpha, i j}(\alpha=1$ or 2$)$. Quenched disorder is parametrized by the set $\left\{\delta J_{\alpha, i j}\right\}$ of random variables. As we are interested in the effects of geometric frustration, we consider antiferromagnetic next-nearest neighbors interactions $J_{2}>0$. As will become clear, our results do not depend on whether the nearest-neighbor interactions are ferromagnetic or antiferromagnetic and, thus, for concreteness, we consider $J_{1}>0$ from now on.

Let us start our analysis reviewing the well-established clean-system $\left(\delta J_{\alpha, i j}=0\right)$ physics $[10,14,15]$ (see also Ref. 16 for a review). For $J_{1}>2 J_{2}$, the classical ground state is a conventional Néel ground state with ordering vector $\mathbf{Q}_{\mathrm{AF}}=(\pi, \pi)$ and ground-state energy $E_{N}=-2 N\left(J_{1}-J_{2}\right)$. On the other hand for $J_{1}<2 J_{2}$, it breaks up into two interpenetrating square sublattices (of unity cell $\sqrt{2} \times \sqrt{2}$ ), each one ordered antiferromagnetically in its own Néel state minimizing the next-nearestneighbor interaction $J_{2}$ as sketched in Fig. 1(a). The corresponding energy is $E_{S}=-2 J_{2} N$ independent of $J_{1}$ and on the polar $\phi$ and azimuthal $\varphi$ angles between two Néel states, and thus, the ground state has an additional $\mathrm{O}(3)$ accidental degeneracy (parametrized by a polar $\phi$ and an azimuthal $\varphi$ angle) known to be lifted by the many sorts of fluctuations: a mechanism known as order by disorder (ObD) [17, 18].

The effects of thermal fluctuations are understood in the following way. Expanding the free energy up to Gaussian fluctuations above the highly degenerate ground state, the resulting $(\phi, \varphi)$-dependent correction to the free energy is

$$
\delta F \propto-T \cos ^{2} \phi
$$



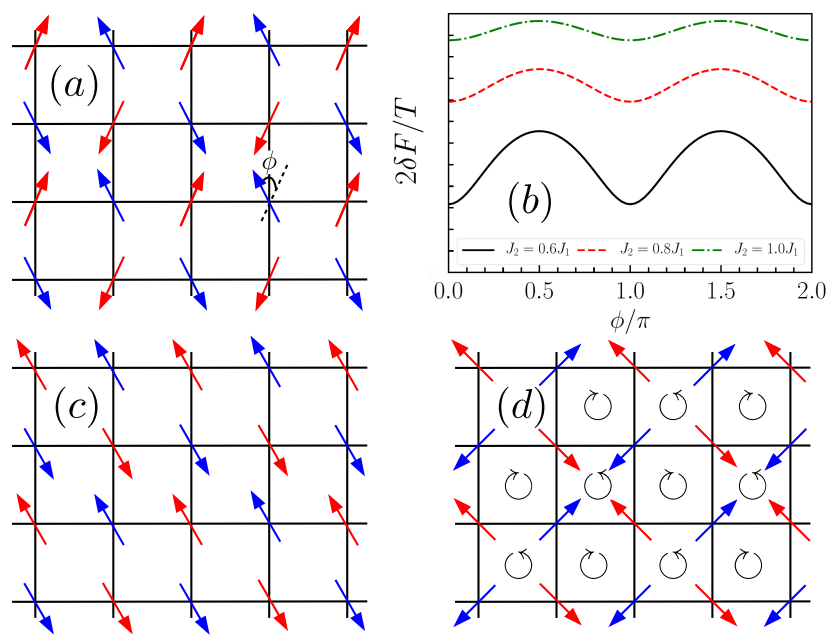

Figure 1. (a) The clean $\left(\delta J_{\alpha, i j}=0\right)$ classical ground state of the Hamiltonian (1) in the regime $J_{1}<2 J_{2}$ : two decoupled antiferromagnetic sublattices (red and blue arrows) with the polar $\phi$ and azimuthal $\varphi$ (not shown) angles parameterizing a nontrivial $\mathrm{O}(3)$ degeneracy. (b) The low- $T$ corrections to the free energy $\delta F$ as a function of the polar angle $\phi$, indicating the selection of the states $\phi=0$ or $\pi$. (c) The collinear (stripe) state $\phi=0$ [ordering wave vector $\left.\mathbf{Q}_{+}=(0, \pi)\right]$. (d) The non-collinear (spin-vortex-crystal) state $\phi=\frac{\pi}{2}, \varphi=0$ with staggered "handness" pattern.

[see Fig. 1(b)] and, therefore, a stripe (collinear) state $(\phi=0$ or $\pi)$ is entropically selected [see Fig. 1(c)]. In other words, the accidental ground-state $\mathrm{O}(3)$ degeneracy is partially lifted by thermal fluctuations remaining a discrete $\mathrm{Z}_{2}$ one: this is the order-by-(thermal)disorder mechanism.

Is the stripe phase stable at low temperatures? If that was the case, then both the continuous $\mathrm{O}(3)$ spin symmetry and the discrete $\mathrm{Z}_{2}$ symmetry would be spontaneously broken. However, the Mermin-Wagner theorem [19] dictates that no continuous symmetry can be spontaneously broken at and below the lower critical dimension $d_{c, \mathrm{MW}}^{-}=2$ at finite $T$ in systems with shortranged interactions. Interestingly, the vestigial (or composite) nematic order associated to the $Z_{2}$ symmetry breaking still takes place at sufficiently low temperatures. Consequently, a continuous finite- $T$ phase transition in the two-dimensional (2D) Ising universality class occurs in the ObD regime $J_{1}<2 J_{2}$. Here, the nematic order parameter is quantified by

$$
\begin{aligned}
\left\langle m_{\|}\right\rangle & =\left\langle N^{-1} \sum_{i} m_{\|, i}\right\rangle, \text { with } \\
m_{\|, i} & =4^{-1}\left(\mathbf{S}_{i}-\mathbf{S}_{j}\right) \cdot\left(\mathbf{S}_{k}-\mathbf{S}_{l}\right)
\end{aligned}
$$

being the local nematic order parameter involving the four spins in the $i$ th plaquette. [Plaquette sites $i k j l$ are arranged counterclockwise as shown in Fig. 3(a)]. Here, $\langle\cdots\rangle$ represents the standard thermal average. The order parameter being $\left\langle m_{\|}\right\rangle>0\left[\left\langle m_{\|}\right\rangle<0\right]$ means a nematic state with ordering vector $\mathbf{Q}_{+}=(0, \pi)\left[\mathbf{Q}_{-}=(\pi, 0)\right]$ and polar angle $\phi=0[\phi=\pi]$.

In Ref. 15, the critical temperature was found to be $T_{c} \approx 0.55 J_{2}$, for $J_{2} \gtrsim 0.9 J_{1}$. Thus, the effective nematic coupling constant is $J_{\|} \propto J_{2}$ in this regime which is in agreement with the field-theory predictions [14].

\section{THE EFFECTS OF QUENCHED DISORDER}

In this section, we describe the effects of quenched disorder (namely, vacancies or bond defects) on the model Hamiltonian (1) in the ObD regime $\left(J_{1}<2 J_{2}\right)$ which will guide our interpretation of the numerical data (see Sec. IV).

We start analyzing how a single and a impurity pair lift the $\mathrm{O}(3)$ ground-state accidental degeneracy. In the following, we show that any finite concentration of impurities yields to non-perturbative effects hindering any paramagnet-nematic phase transition. Finally, combining all these effects in addition to the thermal fluctuations, we characterize the resulting paramagnet.

\section{A. Selection by a single impurity}

In the ObD regime $J_{1}<2 J_{2}$, perturbative approaches $[9,10]$ confirmed by numerical studies [11] predict that a site vacancy selects a set of non-collinear states out of the highly degenerate ground-state manifold, namely the spin-vortex-crystal (SVC) states [see Fig. 1(d)]. The corresponding correction to the groundstate energy due to a density $x \ll 1$ of vacancies is

$$
\delta E \propto x \cos ^{2} \phi,
$$

which favors $\phi=\frac{\pi}{2}$. (Notice that this order-by(quenched)disorder selection is an energetic one.) Therefore, isolated vacancies lift the accidental $\mathrm{O}(3)$ groundstate degeneracy leaving a remaining $\mathrm{O}(2)$ (associated with the azimuthal angle $\varphi$ between the two Néel states). The related vestigial order, named spin-vorticity densitywave (SVDW) order [20], is quantified by the axial vector

$$
\begin{aligned}
& \left\langle\mathbf{m}_{\perp}\right\rangle=\left\langle N^{-1} \sum_{i} \mathbf{m}_{\perp, i}\right\rangle, \text { with } \\
& \mathbf{m}_{\perp, i}=(-1)^{i_{x}+i_{y}} 4^{-1}\left(\mathbf{S}_{i}-\mathbf{S}_{j}\right) \times\left(\mathbf{S}_{k}-\mathbf{S}_{l}\right)
\end{aligned}
$$

being the local SVDW order parameter which involves the four spins of the $i$ th plaquette [as in Eq. (3)], and $i_{x}$ and $i_{y}$ being the coordinates of site $i$.

On the other hand, the selection by a single $J_{1}$-bond defect is quite different. A vertical bond defect $J_{1}+\delta J$, for instance, provides an energy correction to the ground state equal to $\delta E=-\delta J \cos \phi$, and thus, selects the stripe state $\mathbf{Q}_{+}\left(\mathbf{Q}_{-}\right)$if $\delta J$ is positive (negative). Interestingly, the two stripe states extremize the energy correction. This is of no surprise since the stripe states $\mathbf{Q}_{ \pm}$ 
break the vertical/horizontal real-space symmetry. As a result, $J_{1}$-bond disorder acts like a local conjugate field breaking the symmetry between the two stripe states [9].

Finally, let us discuss the effect of a weak $J_{2}$-bond defect. Clearly, it does not lift the ground-state degeneracy since it does not affect the collinear Néel states in each sublattice. We have numerically verified (see Sec. IV E) that a finite concentration of $J_{2}$-bond impurities induces only random-mass disorder which produces only mild effects far from the $J_{1}=2 J_{2}$ transition.

\section{B. Equivalence between site and bond disorder}

The effects of a single site vacancy and a single $J_{1}$-bond impurity are quite different as discussed in Sec. III A. The main reason is because they break different realspace symmetries which selects different states out of the clean ground-state manifold. However, for a finite concentration of impurities, site and bond disorder become equivalent since the symmetries broken by them are the same.

For concreteness, consider for instance two $J_{1}$-bond impurities of same magnitude, one vertical and the other horizontal, meeting at the same site. As the vertical/horizontal symmetry is not locally broken, the $\mathbf{Q}_{ \pm}$ stripe states cannot be selected. Performing numerical energy minimization $[21],{ }^{3}$ we have verified that the selected ground state is the SVC, as expected [see Fig. 2(a)].

We now investigate the robustness of this selection with respect to (i) the anisotropy between vertical and horizontal defects, and with respect to (ii) their distance. Using the energy minimization method, we plot in Fig. 2(b) the ground-state energy (solid line) as a function of the defective vertical coupling amplitude $J_{1}+\delta J^{(\mathrm{v})}$ while the horizontal one is kept missing $\left(\delta J^{(\mathrm{h})}=-J_{1}\right)$. Clearly, the non-collinear SVC state is selected even for a fairly large amount of anisotropy $\delta J^{(\mathrm{v})} \gtrsim 0.4 \delta J^{(\mathrm{h})}$. Beyond that, the SVC ground state smoothly evolves into the stripe state. (The perfect stripe state energy is given by the dashed line.) The inset shows the nematic and SVDW order parameters. Finally, we have verified that the SVC state is energetically selected by any two $J_{1}$ bond impurities regardless of their distance provided that one is vertical and the other is horizontal (and their magnitude are not sufficiently different) as shown in Fig. 2(c).

Given that these two $J_{1}$-bond impurities separately select the two different stripe states, one could suppose that

\footnotetext{
3 We simply sweep the entire lattice and align the spins to their local exchange field. This procedure is guaranteed to find a local energy minima, but it usually struggles to find the global minima in the case of disordered frustrated system, being therefore limited to small system sizes $[12,22,23]$. In order to ensure convergence, we have used many different random initial states, SVC states, and states fed from low-temperatures Monte Carlo simulations.
}

the system would break into two stripe domains (which is the case for Ising spins). However, the domain-wall cost disfavors this configuration and the system prefers the SVC state. Alternatively, we can say that, in order to minimize energy, the domain wall thickness is unbounded (at $T=0$ ) leaving no room for the stripe domains. More importantly, the domain wall exhibits SVC order, the structure factor of which peaks at the two stripe ordering vectors $\mathbf{Q}_{+}$and $\mathbf{Q}_{-}$. As will become clear later, this interpretation is helpful for interpreting the numerical data.

In order to close the equivalence between site and bond disorder, we now show that two site vacancies can also select one of the stripe states. Consider for instance the case of two missing sites one on top of each other. In this case, three vertical and four horizontal $J_{1}$ bonds are missing, and thus, the $\mathbf{Q}_{+}(\phi=0)$ state is energetically favored. Two nearest-neighbor site vacancies are exactly what is needed to locally break the vertical/horizontal symmetry. ${ }^{4}$

\section{Thermal fluctuations and screening}

As shown in Secs. III A and III B, a single or a pair of impurities either select the SVC or the stripe states out of the clean ground-state manifold.

At $T>0$, both orders are melted as they require the spontaneous breaking of the $\mathrm{O}(3)$ (continuous) spinrotation symmetry. The associated nematic vestigial order, on the other hand, is stable at low temperatures and is also entropically selected (see Sec. II). The associated SVDW vestigial order, however, is melted since it also requires the spontaneous breaking of a continuous symmetry. ${ }^{5}$

In the remainder of this section, we discuss the resulting thermal state in the presence of these impurities. Let us start with the trivial case of a single $J_{1}$-bond defect or two nearest-neighbor site vacancies which select, say, the $\mathbf{Q}_{+}$nematic state. As they act as a local nematic field, then at finite $T$ and for finite system size $L$, the nematic order $\left\langle m_{\|}\right\rangle$is always positive. It reaches 1 as $T \rightarrow 0$ and vanishes in the $T \rightarrow \infty$ limit. Upon increasing the system size at finite $T,\left\langle m_{\|}\right\rangle \rightarrow 0$ as $L \rightarrow \infty$ because the free-energy difference between the positive and negative

\footnotetext{
4 Two distant site vacancies in the same row or column do break the global vertical/horizontal real-space symmetry. However, they do not select any of the stripe states as the same amount of vertical and horizontal $J_{1}$ bonds is missing. Thus, the terminology of locally breaking a symmetry means that this is done in a plaquette.

5 One could think that the associated $\mathrm{O}(2)$ symmetry could be quasi-broken in a Berezinskii-Kosterlitz-Thouless transition. However, symmetry considerations indicate that an $\mathrm{O}(3)$ symmetry [related to the axial vector (5)] must be broken before the $\mathrm{O}(2)$ one $[20,24]$, thus, precluding any quasi-long-range SVDW order at finite $T$.
} 

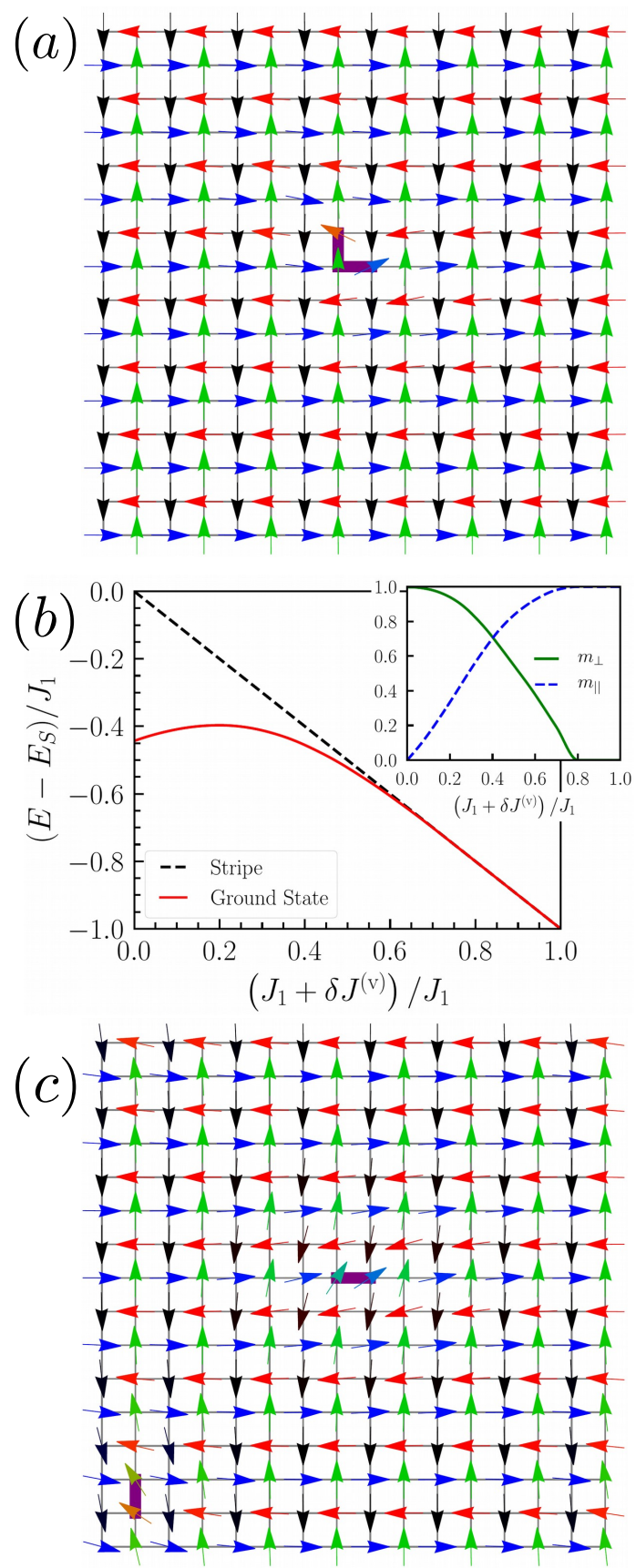

Figure 2. (a) Ground-state configuration obtained by the iterative energy minimization method of the spin configurations. The strong links represent missing nearest-neighbor $J_{1}$ bonds $\left(\delta J^{(\mathrm{v})}=\delta J^{(\mathrm{h})}=-J_{1}\right)$. (b) The ground-state energy (measured with respect to the clean one $E_{S}$ ) (solid line) and the perfect-stripe-state energy $\left[E_{S}-\left(J_{1}+\delta J^{(\mathrm{v})}\right)\right]$ (dashed line) as a function of the bond magnitude $J_{1}+\delta J^{(\mathrm{v})}$ (the magnitude of the other bond is fixed at $\left.\delta J^{(\mathrm{h})}=-J_{1}\right)$. Inset: the corresponding nematic $m_{\|}$and SVDW $m_{\perp}$ order-parameter magnitudes. (c) Same as (a) but with far-apart defects. The system size is $L=16$ and periodic boundary conditions are considered. The value $J_{2}=0.55 J_{1}$. nematic states is non-extensive. Thus, the $\operatorname{limits} T \rightarrow 0$ and $L \rightarrow \infty$ do not commute.

Let us now discuss the more interesting case of a singlesite vacancy which selects the SVC order. There are two mechanisms confining that order in a region of size $\xi_{\perp}$ around the impurity: the competing nematic order and the thermal fluctuations. At finite temperatures (below the clean critical temperature $T_{c}$ ), the impurity generates a perturbation inside the nematic state which cannot be propagated beyond the nematic correlation length $\xi_{\|}$. Thus, $\xi_{\perp}$ is bounded by $\xi_{\|}$. Evidently, SVC and SVDW orders are also bounded by the thermal correlation length $\xi_{\mathrm{MW}}$ of a continuous symmetry order parameter which, at low temperatures, is $\propto e^{\rho_{\perp} / T}$, with $\rho_{\perp}$ being a related stiffness. (The exponentially large $\xi_{\mathrm{MW}}$ is characteristic of the lower critical dimension of the problem $d_{c, \mathrm{MW}}^{-}=2$.) Therefore, $\xi_{\perp} \propto \min \left\{\xi_{\mathrm{MW}}, \xi_{\|}\right\}$.

An analogous effect would occur for two bond defects provided that their distance is smaller than $\xi_{\perp}$, that one is vertical and the other horizontal, and that their magnitudes are similar. If their distance is greater than $\xi_{\perp}$, the SVDW domain wall between them is entropically disfavored and the system will be in either one of the equally equivalent nematic states.

\section{The effects of disorder-induced random nematic fields}

In this section we show the impossibility of a phase transition into a nematic phase in the presence of any finite concentration of impurities in $d \leq 2$. The proof is by reductio ad absurdum. We assume the existence of the transition and arrive in a contradiction.

Assuming that the transition exists, the symmetry of nematic order parameter (3) dictates that a spontaneous symmetry-breaking transition is in the unfrustrated 2D Ising universality class $[11,14,15,20]$. Therefore, the nematic transition can be described by the 2D Ising model with generic short-range ferromagnetic interactions with the Ising variables representing the two nematic states $\mathbf{Q}_{ \pm}$. As discussed in Secs. III A and III B, $J_{1}$-bond defects and nearest-neighbor vacancy pairs act as nematic fields locally breaking the symmetry between the $\mathbf{Q}_{ \pm}$states. Therefore, in the presence of a finite concentration of impurities we must furnish the effective Ising model with additional random fields and random couplings. The lower critical dimension of this model is known to be $d_{c, \| \mathrm{RF}}^{-}=2[5,25-27]$. Consequently, there is no phase transition in $d \leq 2$. This ends our proof.

We recall that, for $J_{1}$-bond disorder, these conclusions were previously obtained in Ref. 9 using conventional perturbative field-theory methods. For site disorder, it was suggested that the transition exists (see also Ref. 11).

In the case of anisotropic disorder, ${ }^{6}$ the mean value of

${ }^{6}$ By anisotropic disorder we mean those situations in which the 
the random fields is finite and hence the corresponding nematic state is globally favored. In this case, $\left\langle m_{\|}\right\rangle$is finite at any temperature.

In the case of isotropic disorder, the random fields have zero mean and, thus, the nematic order parameter is always vanishing. At low temperatures, the system is broken into domains of different nematic order of typical size $\xi_{\| \operatorname{RF}} \propto e^{\tilde{J}^{2} / \sigma_{\tilde{h}}^{2}}$ (up to power-law corrections) [2527]. Here, $\tilde{J}$ is the mean value of the effective nematic coupling constants $J_{\|}$(proportional to $J_{2}$ in the regime $J_{2} \gtrsim J_{1}$ ), and $\sigma_{\tilde{h}}^{2}$ is the variance of the effective random fields $\tilde{h}$. In the case of low density of bond defects $0<x \ll 1, \sigma_{\tilde{h}}^{2} \propto x(1-x)$. Likewise, for a low density $x$ of site vacancies, $\sigma_{\tilde{h}}^{2} \propto x^{2}\left(1-x^{2}\right)$. The fact that $\xi_{\| \mathrm{RF}}$ is exponentially large in $1 / \sigma_{\tilde{h}}^{2}$ is a consequence of the system being at the lower critical dimension $d=d_{c, \| \mathrm{RF}}^{-}$. It also points out to the huge difference between the domain sizes formed by bond and site defects in the limit of small defect concentrations $x$.

In the Appendix, we apply these simple arguments to show the disorder-induced generation of random axes in easy-plane pyrochlores.

\section{E. Disorder-induced dipolar random fields}

We now show that the spin-vortex-crystal state is unstable in the presence of any finite concentration of impurities in $d \leq 2$ even at $T=0 .{ }^{7}$ The reason is akin to the instability of the nematic order discussed in Sec. (IIID): impurities-induced random fields. As in the nematic case, it is also related to a real-space symmetry broken by the ordered state: the inversion symmetry. However, the random fields are non-local exhibiting a dipolar texture.

This result is a direct consequence of the theory developed in Ref. 12 where it was shown that any amount of generic bond disorder destroys any non-collinear order. As a consequence of the symmetry arguments of Sec. III B, we extend this result to site disorder as well (see below). Finally, there is another important difference with respect to the stripe/nematic case. The vestigial SVDW is perturbatively stable against disorder at $T=0$. This does not contradict the results of Ref. 12 since the SVDW order is collinear.

We start our analysis by numerically verifying that a single $J_{1}$-bond defect produces a dipolar spin texture [12]

$$
\delta \theta(\mathbf{r})=\alpha \delta J \frac{\hat{e} \cdot \mathbf{r}}{r^{d}}
$$

global vertical/horizontal isotropy is broken such as, for instance, the cases in which the disorder averages of $\delta J_{1}^{(\mathrm{v})}$ and of $\delta J_{1}^{(\mathrm{h})}$ are different, or when the density of horizontal and vertical vacancy pairs are different.

7 This is a pertinent question even in the realistic case of quantum spins. For a sufficiently high concentration of site vacancies, the SVC state may percolate.
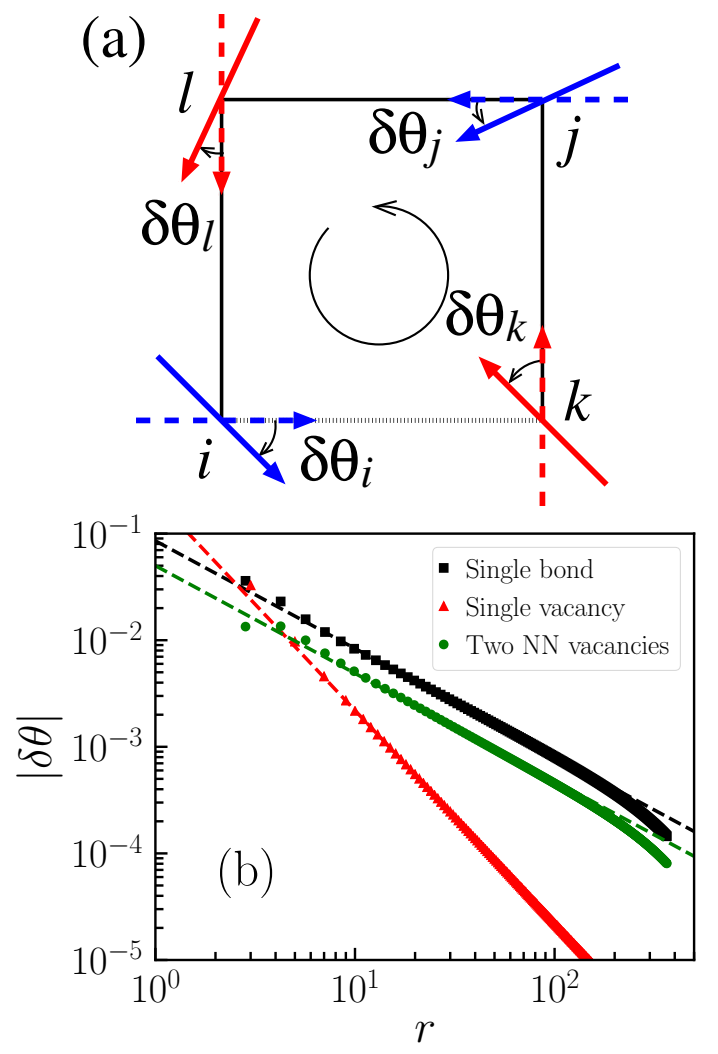

Figure 3. (a) The spin rotation field on a plaquette induced by a strong $(\delta J>0) J_{1}$-bond impurity (dotted line). (Dashed arrows represent the unperturbed state.) (b) The spin-rotation field magnitude $|\delta \theta|$ plotted as a function of distance $r$ from the defect (see text for details) for the cases of a $J_{1}$-bond vacancy $\left(\delta J=-J_{1}\right)$ (black squares), a single vacancy (red triangles) and a pair of nearest-neighbor vacancies (green circles). We have used $J_{2}=0.55 J_{1}$ and fixed $K=0.05 J_{1}$. We used system sizes up to $L=2^{10}$ with periodic boundary conditions. The dashed lines are power-law fits $\propto r^{-1}$ and $\propto r^{-2}$ in the range $10 \lesssim r \lesssim 10^{2}$. Deviations at large $r$ are due to finite-size effects. We have obtained similar results (not shown) for system size $L=400, K=0.1 J_{1}$, and $J_{2} / J_{1}=0.55,0.6$ and 0.8 .

on the spin-vortex-crystal state [see Fig. 3(a)]. Here, $d=$ $2, \delta \theta(\mathbf{r})$ is the in-plane impurity-induced angle deviation (with respect to the spin-vortex-crystal state) of the spin at position $\mathbf{r}$ measured with respect to the center of the defective bond, the unity vector $\hat{e}$ is the direction of the defective bond $(\hat{x}$ or $\hat{y})$, and $\alpha \equiv \alpha\left(J_{1}, J_{2}\right)$ is a constant the sign of which ensures that the two spins connected by the defective bond align more (less) anti-parallel for $\delta J>0(\delta J<0)$. (Notice therefore that the local random field lies within the SVC plane and is perpendicular to the local spin order.)

In order to stabilize the non-collinear SVC state in a clean sample, we supplement the Hamiltonian (1) with a positive biquadratic term $K \sum_{\langle i j\rangle}\left(\mathbf{S}_{i} \cdot \mathbf{S}_{j}\right)^{2}[10,20]$. The $T=0$ resulting spin configuration is obtained via numerical energy minimization [21]. Convergence is confirmed 
by starting from many different initial random states. In all cases the final configuration is the same and remains coplanar. In Fig. 3(b) we plot $|\delta \theta|$ as a function of $r$ where $\mathbf{r}=r \hat{e}$. We have verified the induced dipolar spin texture (6) by inspection of our data (not shown). ${ }^{8}$ This result can be derived analytically through linear response theory in any dimension $d$ as shown in details in Ref. 12. Here, we only mention the reason for such. The fact that the SVC state breaks the inversion symmetry makes the distortion field $\delta \theta(\mathbf{r})$ an odd function with respect to the inversion $\mathbf{r} \rightarrow-\mathbf{r}$. Therefore, the spin texture is $p$-wave like which is communicated to the rest of the system by in-plane Goldstone modes and, thus, decays as $\sim 1 / r[28]$.

The equivalence between bond and site disorder is confirmed in Fig. 3(b) where $|\delta \theta|$ is plotted for the case of a nearest-neighbor vacancy pair. (Here, $\mathbf{r}$ is measured from the center of the vacancy pair and $\hat{e}=\hat{y}$ or $\hat{x}$ is the direction perpendicular to the vacant sites.) Again, we verify the induced dipolar spin texture (6). (In contrast, a single vacancy induces a quadrupolar texture which decays $\sim 1 / r^{2}[11]$.)

Having verified the dipolar spin texture (6) induced by a single-bond impurity or a nearest-neighbor vacancy pair, it is now straightforward to show the instability of the SVC order against any finite concentration of impurities in $d \leq 2$. Averaging over the disorder configurations (denoted by $[\cdots]$ ), the mean angle deviation at site $j$ vanishes because disorder is globally isotropic: $\left[\delta \theta_{j}\right] \propto \sum_{m} \delta J_{m} \frac{\hat{e}_{m} \cdot \mathbf{r}_{j, m}}{r_{j, m}^{d}}=0$, where $\mathbf{r}_{j, m}$ is the position of site $j$ with respect to the $m$ th impurity. The variance, on the other hand, behaves quite differently: $\left[\delta \theta_{j}^{2}\right] \propto \sum_{m, n} \delta J_{m} \delta J_{n} \frac{\hat{e}_{m} \cdot \mathbf{r}_{j, m}}{r_{j, m}^{d}} \frac{\hat{e}_{n} \cdot \mathbf{r}_{j, n}}{r_{j, n}^{d}} \sim \int r^{1-d} \mathrm{~d} r$ is infrared divergent for $d \leq d_{c, \perp \mathrm{RF}}^{-}=2$ precluding any SVC order. At the lower critical dimension of the problem, it was shown that SVC order is confined in a domain of temperature-independent typical size $\xi_{\perp R F} \propto e^{\rho_{\|}^{2} / \sigma_{\delta J}^{2}}$, where $\rho_{\|}$is a related stiffness and $\sigma_{\delta J}^{2}$ is the variance of $\delta J_{1}[12]$. The resulting ground state is a SVC spin-cluster glass.

As expected, we verified that disorder on the $J_{2}$ bonds does not induce dipolar random fields.

How about the stability of vestigial SVDW order? At $T=0$ in a coplanar state, the local SVDW order (5) reduces to a scalar and therefore the result of Ref. 12 does not apply. Let us consider the effects of the dipolar spin texture (6) on the SVDW order. Far from the impurity, the change in the local order parameter is $\delta m_{\perp}(\mathbf{r}) \propto-\left(1+d(d-2)(\hat{e} \cdot \hat{r})^{2}\right)\left(\delta J r^{-d}\right)^{2}$, which vanishes sufficiently fast as $r \rightarrow \infty$. Consequently, the mean deviation $\left[\delta m_{\perp}\right] \propto\left[(\delta J)^{2}\right]$ is finite and, more im-

\footnotetext{
8 A careful reader will notice in Fig. 2(c) a non-dipolar spin texture near the impurities. We emphasize that the dipolar character (6) dominates only at larger distances invisible to the naked eye.
}

portantly, because $\delta m_{\perp}$ is isotropic at $d=2$, the corresponding variance vanishes up to $\mathcal{O}(\delta J)^{4}$. This strongly suggests that SVDW order is perturbatively stable at $d=2$. Our numerics (see Sec. IV F) support this scenario. At weak disorder, the ground-state spin configuration remains coplanar with vanishing SVC order and finite SVDW order. Upon increasing disorder, the spin configuration becomes non-coplanar and the SVDW order is destroyed.

\section{F. Phase diagram}

As discussed in the previous sections, in the presence of generic disorder and in the ObD regime $\left(J_{1}<2 J_{2}\right)$ the model system (1) is a always a paramagnet at $T>0$ and a spin-vortex-crystal cluster-spin glass at $T=0$ with finite spin-vorticity density-wave vestigial order in the regime of weak disorder.

It is noteworthy that the paramagnet is not uninteresting. It is broken into spin clusters locally exhibiting nematic order. Therefore, it is polarized in the nematic spin-cluster-glass order. With respect to the nematic order, this paramagnet is very much similar to that of the Ising model in a random field which is not a glass [29]. However, with respect to susceptibilities they are quite different because (i) the nematic effective coupling constant is temperature dependent (due to the entropic selection) and (ii) the domain walls in our case have high SVDW susceptibility. As a consequence, the domain-wall thickness is temperature dependent yielding nontrivial nematic and SVDW susceptibilities. An explicit discussion on this issue involving also the glassy properties is given in Sec. IV D.

\section{NUMERICAL SIMULATIONS}

In this section we report our Monte Carlo (MC) simulations of the system Hamiltonian (1) and our numerical zero-temperature energy minimization.

\section{A. Technical details}

In our MC code, we have used both the Heat Bath [30] and the Microcanonical [31] local update algorithms. Precisely, we employ 5 Heat Bath steps followed by 5 Microcanonical ones (1 MC step means 1 random sweep over the entire lattice), which proved to be an optimal choice for equilibration. After these $10 \mathrm{MC}$ steps, we finally perform a parallel-tempering step. We define a temperature grid such that the probability of swapping adjacent configurations is independent of the temperature values and is sufficiently high so as to ensure frequent exchanges [32]. We typically perform $5 \times 10^{5} \mathrm{MC}$ steps to reach equilibrium and $M=5 \times 10^{5} \mathrm{MC}$ steps to 
take averages. ${ }^{9}$

As usual in MC studies, the thermal average is replaced by the MC average, i.e.,

$$
\langle\mathcal{O}\rangle \rightarrow M^{-1} \sum_{t=1}^{M} \mathcal{O}(t),
$$

where $\mathcal{O}(t)$ is the value of the observable $\mathcal{O}$ at the $t$ th Monte Carlo step (after equilibration).

When studying disordered systems, we also average an observable over $N_{d}=600$ different disorder realizations (denoted by $[\cdots]$ ), namely, $[\langle\mathcal{O}\rangle]=N_{d}^{-1} \sum_{k=1}^{N_{d}}\langle\mathcal{O}\rangle_{k}$, where $\langle\mathcal{O}\rangle_{k}$ is the MC average of $\mathcal{O}$ in the $k$ th disorder realization.

In addition to the nematic (3) and SVDW (5) order parameters, we also study the corresponding susceptibilities, the nematic Binder cumulant, and the specific heat, respectively given by

$$
\begin{aligned}
\chi_{a}^{\alpha} & =\frac{N}{T}\left[\left\langle\left(m_{a}^{\alpha}\right)^{2}\right\rangle-\left\langle\left|m_{a}^{\alpha}\right|\right\rangle^{2}\right], \\
U_{\|} & =1-\frac{1}{3}\left[\frac{\left\langle m_{\|}^{4}\right\rangle}{\left\langle m_{\|}^{2}\right\rangle^{2}}\right], \\
c_{v} & =(N T)^{-1}\left[\left\langle E^{2}\right\rangle-\langle E\rangle^{2}\right],
\end{aligned}
$$

where $a=\|, \perp$, respectively, refers to the nematic and SVDW orders, $\alpha, \beta=x, y, z$ are the order-parameter components (not applicable to the nematic case), and $E$ is the spin configuration energy (1).

In addition, we also have studied the nematic and SVDW Edwards-Anderson order parameters defined as $[33,34]$

$$
\left[\left\langle m_{\mathrm{EA}, a}\right\rangle\right]=\left[\left\langle\sqrt{N^{-1} \sum_{\alpha, \beta}\left|\sum_{i} m_{a, i}^{\alpha(1)} m_{a, i}^{\beta(2)}\right|^{2}}\right\rangle\right]
$$

where the super-indices (1) and (2) denotes the two simulated replicas, i.e., we independently simulate two copies of the system (same disorder configuration), and thus, $\left\langle m_{a, i}^{\alpha(1)} m_{a, i}^{\beta(2)}\right\rangle=\left\langle m_{a, i}^{\alpha(1)}\right\rangle\left\langle m_{a, i}^{\beta(2)}\right\rangle$. The corresponding spin-glass susceptibility $\chi_{\mathrm{EA}, a}$ is the corresponding thermal fluctuation magnitude of $m_{\mathrm{EA}, a}$ quantified by (8) with $m_{a}^{\alpha}$ replaced by $m_{\mathrm{EA}, a}$.

\footnotetext{
9 We think it is worthy to mention that we have performed a MC study without parallel tempering and obtained the same results. (Although it needs $5 \times 10^{5} \mathrm{MC}$ steps to reach equilibrium and $M=1.5 \times 10^{6} \mathrm{MC}$ steps to take averages in order to obtain equivalent error bars.) We interpret this result as the absence of a slowing down related to any glass-like behavior.
}
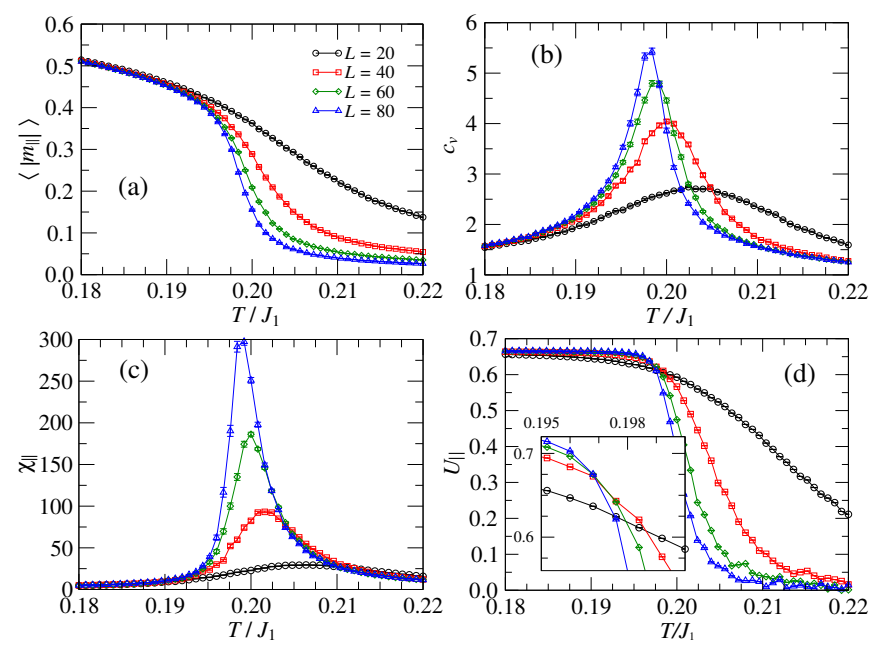

Figure 4. The MC results for clean system and $J_{2}=0.55 J_{1}$. (a) The modulus of the nematic order parameter, (b) the specific heat, (c) the nematic susceptibility, and (d) the Binder cumulant as a function of the temperature for several system sizes. The inset shows a magnification around the crossing points signaling the location of the transition temperature $T_{c} \approx 0.197(1) J_{1}$.

\section{B. The clean system}

In order to benchmark our codes, we review the impurity-free nematic phase transition in the $\mathrm{ObD}$ regime where we fix $J_{2}=0.55 J_{1}$.

In Fig. 4 we plot the specific heat, the absolute value of the nematic order parameter, the nematic susceptibility and Binder cumulant as a function of the temperature for various system sizes.

Clearly, these observables exhibit the usual temperature dependence of a continuous phase transition. In particular, the Binder cumulant tends to $\frac{2}{3}$ for $T<T_{c}$ and to 0 for $T>T_{c}$ in the thermodynamic limit. For finitesize systems, extrapolating the crossings of different sizes (not shown) allows us to estimate the critical temperature to $T_{c} \approx 0.197(1) J_{1}$. The standard finite-size scaling analysis $[35,36]$ is shown in Fig. 5 from which we obtain $\nu=1.00(6)$ and $\gamma=1.72(5)$, compatible with the 2D Ising universality class $(\nu=1$ and $\gamma=7 / 4)$ and the earlier estimate reported in Ref. 15.

\section{Spatially anisotropic disorder}

In this section we study the effects of dilution of horizontal $J_{1}$ bonds and of horizontal nearest-neighbor site pairs. We do not find any hint of a phase transition. Instead, the system is always polarized in the $\mathbf{Q}_{+}\left(\mathbf{Q}_{-}\right)$ nematic state when of bond (site) dilution.

We start by reporting the effects of a single $J_{1}$-bond impurity. We have verified (not shown for the sake of brevity) that, for low temperatures and small sample sizes, the nematic order parameter (3) is always posi- 
tive in agreement with the predictions of Sec. (III A). (Recall that $m_{\|}$averages to zero in the clean system.) Accordingly, as the temperature is raised and the system size enlarged, the difference between the $\mathbf{Q}_{ \pm}$states diminishes and so does the order-parameter mean value.

We now report our study for the case of a finite fraction of impurities. Figure 6 is equivalent to Fig. 4 but with $2 \%$ of horizontal $J_{1}$ bonds missing. Clearly, there are no indications of singularities as the system size increases. More importantly, there is no hint of crossings in the Binder cumulant, implying no phase transition even above the clean critical temperature $T_{c} \approx 0.19 J_{1}$. Notice also that the order parameter is always finite (and positive) for any finite temperature in agreement with our expectations (see Sec. IIID). Finally, another support of this conclusion is the strong indication of the Binder cumulant saturating to $\frac{2}{3}$ in the thermodynamic limit for all temperatures.

Finally, we report that a completely similar study was conducted when $1 \%$ of the lattice sites are diluted in such a way that they appear in pairs of horizontal nearest neighbors. We report that completely analogous results as in Fig. 6 were found and, for that reason, we do not to show them here. Instead, we present a complementary result: the density plot of the local nematic order parameter for a typical disorder configuration (see the top panel of Fig. 7). Clearly, the $\mathbf{Q}_{-}$-state is selected. We have verified that this is the case in all temperatures. For comparison, in the bottom panel we show a typical density plot for the case of $0.5 \%$ of vertical $J_{1}$-bond dilution. Apart from the localized spin texture around the defects, these plots are essentially the same.

\section{Spatially isotropic disorder}

We now report on the more subtle case of isotropic disorder in which the global (statistical) vertical/horizontal real-space symmetry is preserved. Specifically, we consider the cases of uncorrelated $J_{1}$-bond dilution. [We have also considered site dilution and obtained similar results but with much larger finite-size effects since $\xi_{\| \mathrm{RF}}$ are considerably larger (see Sec. IIID).]

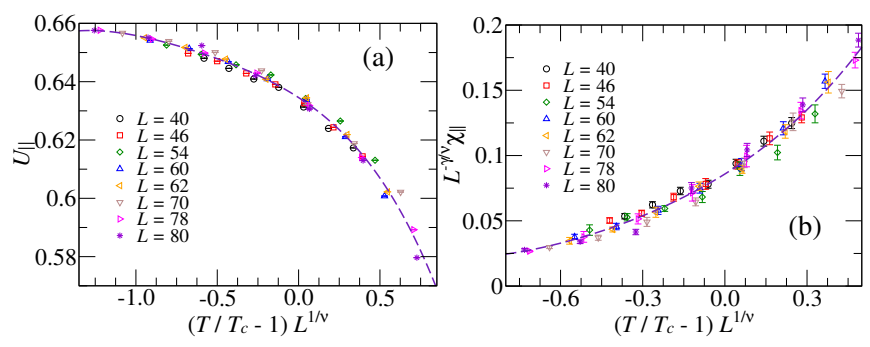

Figure 5. The finite-size scaling replot of (a) the Binder cumulant and (b) the nematic susceptibility (right) data of Fig. 4. The lines are simple quartic best fits representing the corresponding scaling functions.
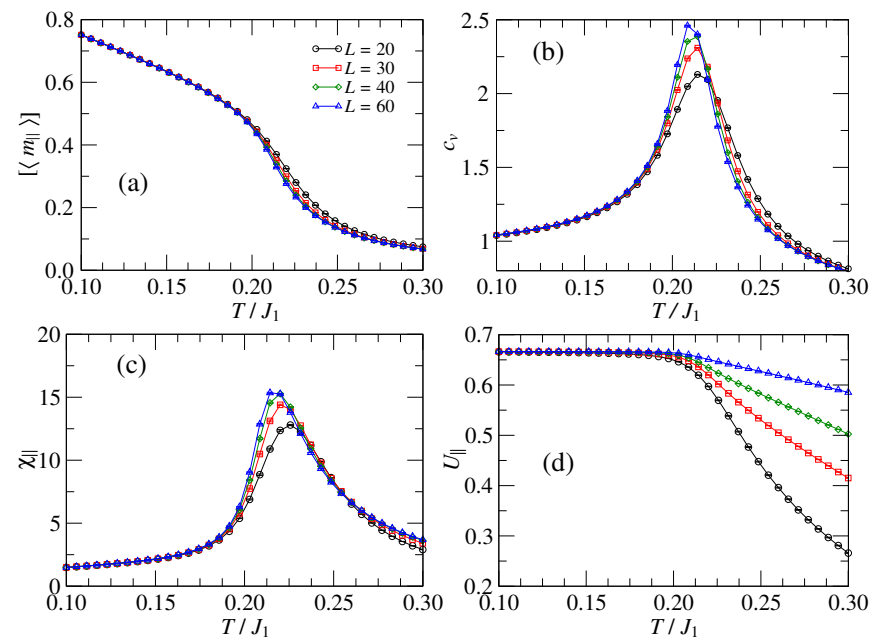

Figure 6. The MC results for the case of $2 \%$ dilution of horizontal $J_{1}$ bonds where $J_{2}=0.55 J_{1}$. (b) The specific heat, (a) the nematic order parameter and (c) the corresponding susceptibility, and (d) the Binder cumulant as a function of the temperature for several system sizes.

In agreement with the arguments of Sec. III, we have verified that there is no phase transition and the system is always a paramagnet. This is illustrated in Fig. 8 where the specific heat, the nematic Binder cumulant, order parameter and susceptibility are plotted as a function of the temperature for samples of many sizes when $2 \%$ of the $J_{1}$ bonds are isotropically diluted. The SVDW order parameter and susceptibility are also shown. Notice the striking difference with respect to the clean case. There is no hint of singularities in any observable. In addition, both the nematic order parameter and the Binder cumulant decrease with increasing the system size for all temperatures. This is a strong indication of a state without nematic long-range order. As expected, the SVDW order parameter behaves likewise. Notice it vanishes slowly at lower temperatures since the associated correlation length $\xi_{\perp}$ is exponentially large as $T \rightarrow 0$.

We now present further details on the glassy features of the resulting paramagnet. In Fig. 9, the nematic Edwards-Anderson order parameter (11) and the corresponding susceptibility for $2 \%$ of diluted $J_{1}$ bonds are plotted. Clearly, $\left[\left\langle m_{\mathrm{EA}, \|}\right\rangle\right]$ is finite in the entire temperature range studied. Notice that, similar to the randomfield Ising model [29], this is not a nematic cluster-spin glass but a paramagnet polarized in that glassy order since $\chi_{\mathrm{EA}, \|}<\chi_{\|}$. The SVDW Edwards-Anderson order parameter and the corresponding susceptibility are also plotted. As expected, $\left[\left\langle m_{\perp}\right\rangle\right]$ [Fig. 8(e)] and [ $\left.\left\langle m_{\mathrm{EA}, \perp}\right\rangle\right]$ diminish very slowly as the system size increases. We have studied the cases of 10\% (see Fig. 10) and 20\% (not shown) of $J_{1}$-bond dilution and observed qualitatively similar results.

As mentioned in Sec. III F, the many competing orders yields this an interesting paramagnet as evident from the non-monotonic behavior of $\chi_{\perp}$ in Fig. 10(d). In the fol- 
lowing, we discuss about the usual and glassy nematic and SVDW susceptibilities in a qualitative level. A full quantitative study is beyond the scope of this work.

Let us start with the nematic susceptibility. In analogy with the random-field Ising model, one could naively expect $\chi_{\|}$to be round-peaked at a temperature $T_{\|}^{*}$ proportional to the clean paramagnet-nematic transition temperature $T_{c}$ as this is the relevant energy scale for the nematic fluctuations. The weaker the disorder, the higher and sharper is the peak (as larger is the typical size of the nematic clusters $\xi_{\| \mathrm{RF}}$ ). However, unlike the Ising model, to the left of of this peak the nematic energy scale $J_{\|}$ is temperature dependent and, thus, we expect $\chi_{\|}$to remain high at lower temperatures as can be seen in our MC data Figs. 8 and 10. With respect to the EdwardsAnderson nematic susceptibility, also in analogy with the random-field Ising model, we expect $\chi_{\mathrm{EA}, \|}$ to be similar to but bounded by $\chi_{\|}$, in agreement with our numerics.

Let us now discuss the more subtle SVDW susceptibil-

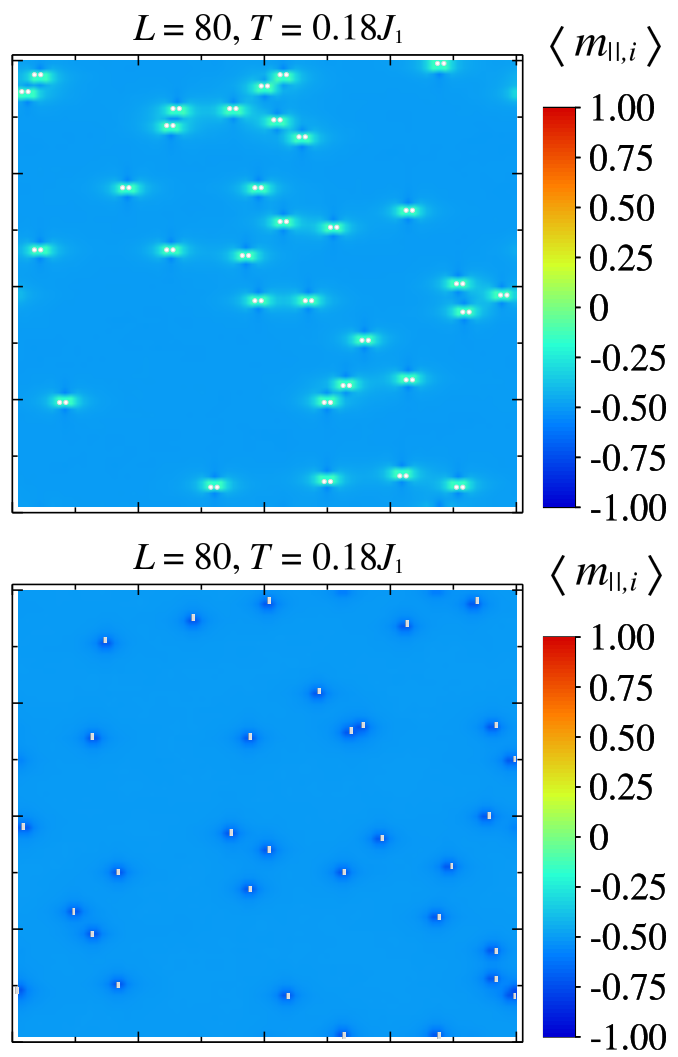

Figure 7. A typical density plot of the local nematic order parameter $\left\langle m_{\|, i}\right\rangle$ [see Eq. (3)] for a lattice of size $L=80$, temperature $T=0.18 J_{1}$ and $J_{2}=0.55 J_{1}$. The light dots (top) and light ticks (bottom) show the position of the missing sites (1\% on average) and vertical bonds ( $0.5 \%$ on average), respectively. Notice that the site impurities always appear in pairs of horizontal nearest neighbor sites locally favoring the

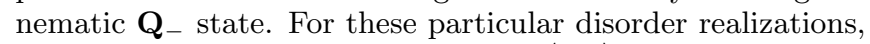
the nematic order parameter averages $\left\langle m_{\|}\right\rangle=-0.486(1)$ (site dilution) and $-0.521(1)$ (bond dilution).
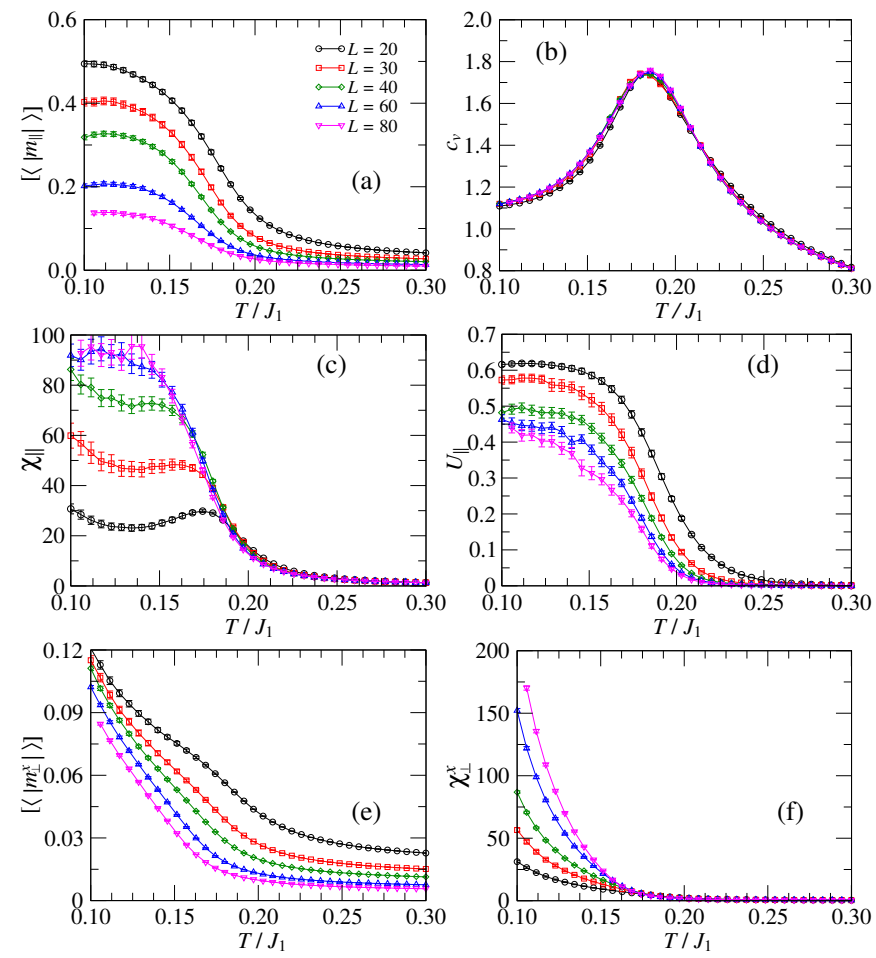

Figure 8. The MC results when $2 \%$ of $J_{1}$ bonds are isotropically diluted and $J_{2}=0.55 J_{1}$. The (b) specific heat, the (d) nematic Binder cumulant, the (a) nematic and (e) SVDW order parameters, and their corresponding susceptibilities [(c) and (f), respectively], all as a function of the temperature and for several system sizes. [We verified that $m_{\perp}^{y, z}\left(\chi_{\perp}^{y, z}\right)$ is statistically identical to $m_{\perp}^{x}\left(\chi_{\perp}^{x}\right)$.]

ity starting our analysis from the low- $T$ limit. We expect both $\chi_{\perp}$ and $\chi_{\mathrm{EA}, \perp}$ to diverge as $T \rightarrow 0$ since the associated correlation length $\xi_{\perp}$ diverges. ${ }^{10}$ This is only possible because the domain walls are sufficiently thick at the lowest temperatures occupying most of the bulk. (Thus, the greater the impurity concentration the greater $\chi_{\perp}$ and $\left.\chi_{\mathrm{EA}, \perp}.\right)$ As temperature increases both $\chi_{\perp}$ and $\chi_{\mathrm{EA}, \perp}$ decrease due to (i) thermal fluctuations and to (ii) the growth of the nematic domains. Beyond $T_{\|}^{*}$, the nematic domains decrease again giving further room to the SVDW domain wall, and thus, $\chi_{\perp}$ develops inflection points (or even a local minimum) near $T_{\|}^{*}$. The same effect is expected on $\chi_{\mathrm{EA}, \perp}$, however, less intensively due to the high temperature.

We end this section by studying more quantitatively the temperature dependence of the size of nematic domains. In Fig. 11, we plot the nematic order parameter distribution $P\left(m_{\sigma}\right)$ for $2 \%$ dilution of $J_{1}$ bonds. It is built as a normalized histogram of the entire MC time series

10 As discussed in Sec. III E, the SVDW order is perturbatively stable against disorder and thus, $\xi_{\perp}$ is not bounded by the dipolar random field correlation length $\xi_{\perp \mathrm{RF}}$. 

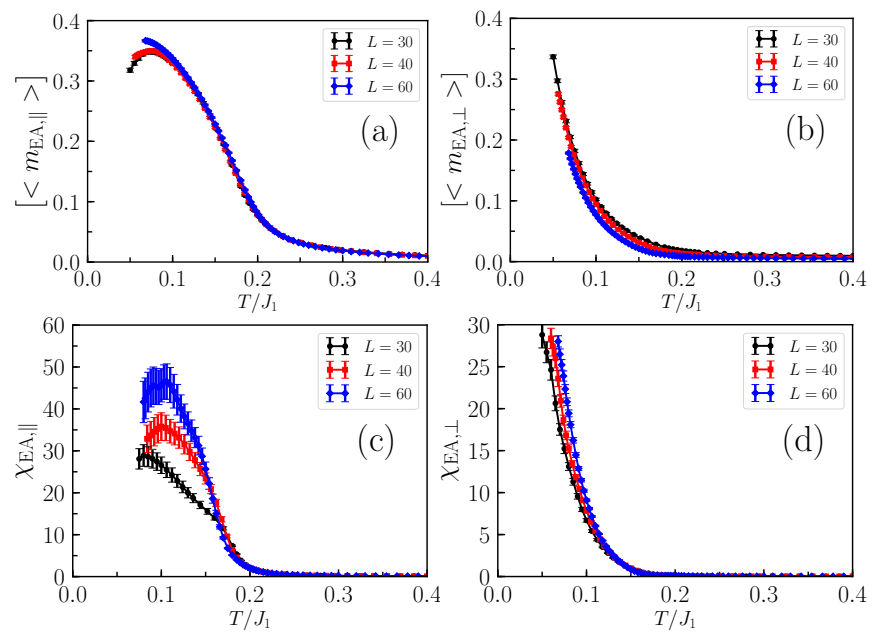

Figure 9. The Edwards-Anderson nematic and SVDW order parameters and the corresponding susceptibilities as a function of the temperature for the same parameters as in Fig. 8.
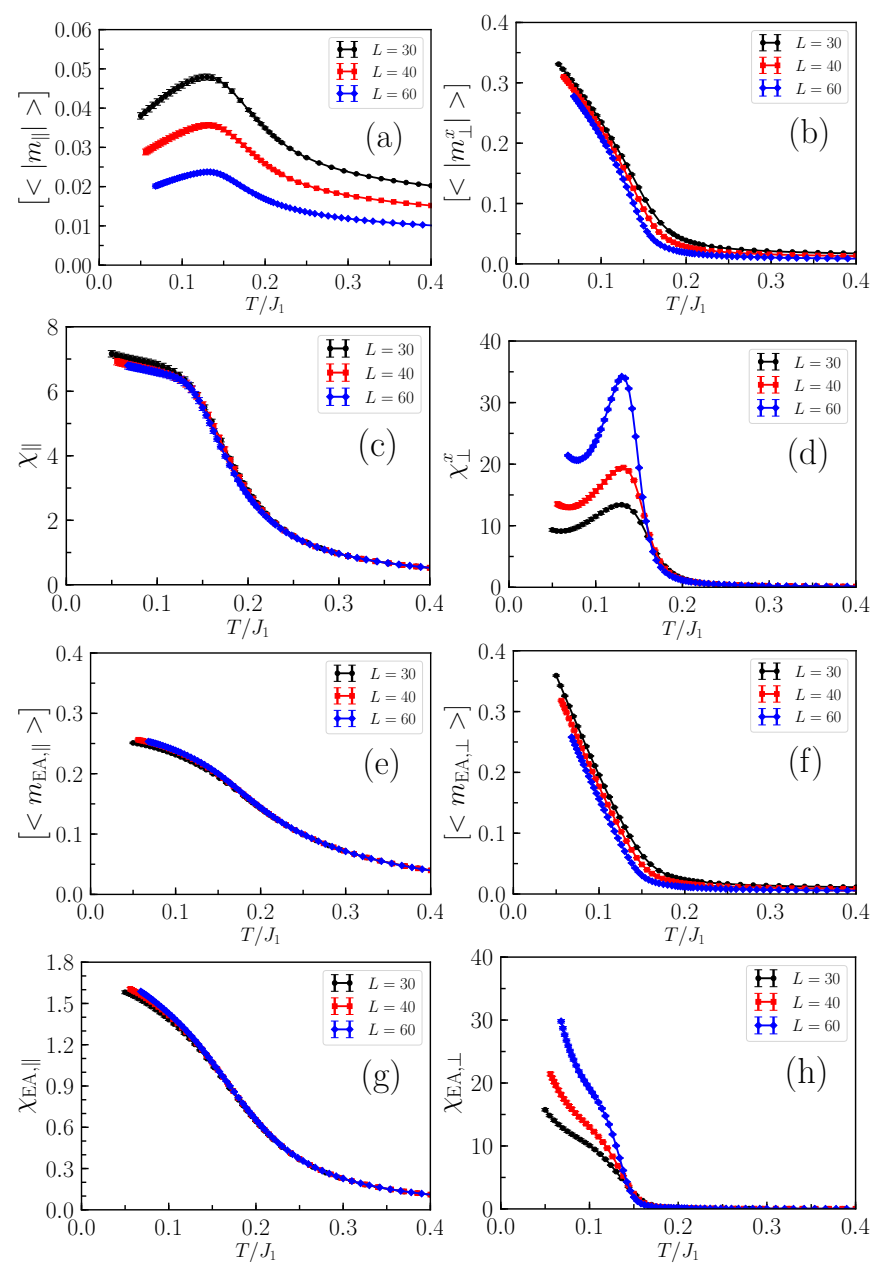

Figure 10. The conventional and the Edwards-Anderson nematic and SVDW order parameters and the corresponding susceptibilities as a function of the temperature for $10 \%$ of isotropically dilution of $J_{1}$ bonds. [We verified that $m_{\perp}^{y, z}$ $\left(\chi_{\perp}^{y, z}\right)$ is statistically identical to $m_{\perp}^{x}\left(\chi_{\perp}^{x}\right)$.]
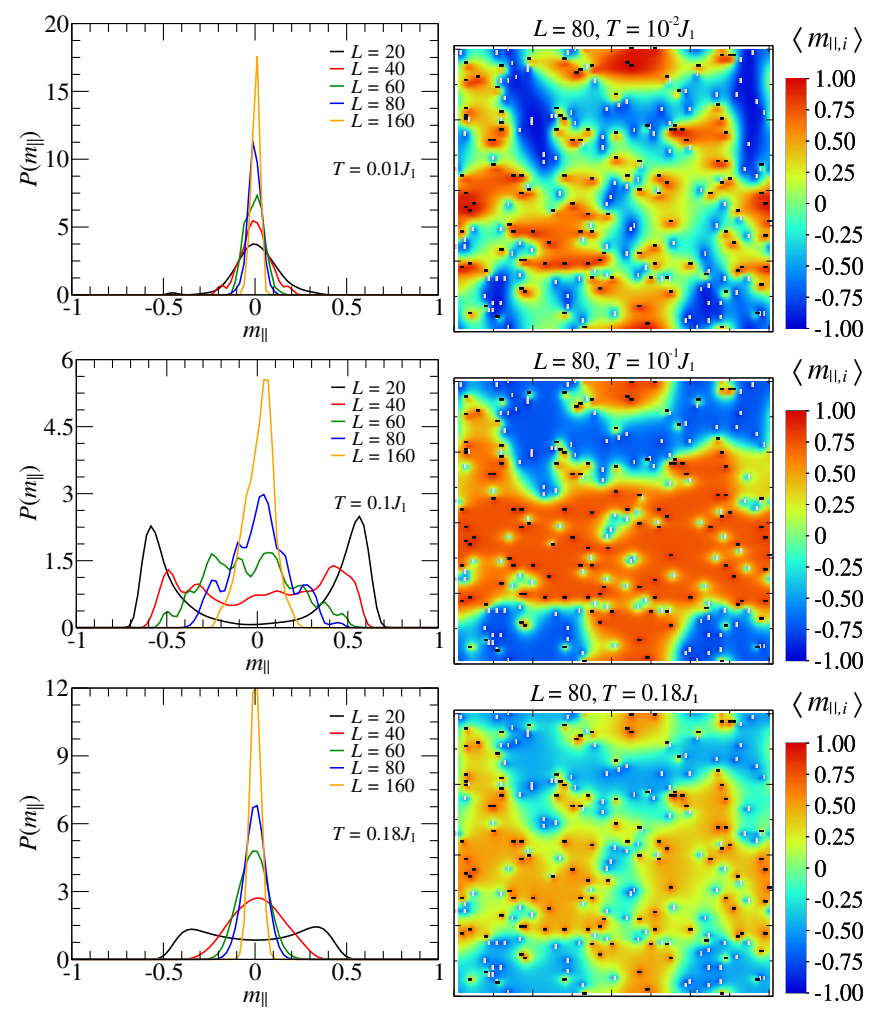

Figure 11. (Left panels) The nematic order-parameter normalized histogram $P\left(m_{\|}\right)$for different system sizes and temperatures and (right panels) the density of the local nematic order $\left\langle m_{\|, i}\right\rangle$ for a typical disorder realization. Here, the vertical (light) and horizontal (dark) ticks represent the missing $J_{1}$ bonds ( $2 \%$ on average).

$m_{\|}(t)$ for all the disorder realizations. We have studied the temperatures $T=10^{-2} J_{1}, 10^{-1} J_{1}$ and $0.18 J_{1}$. For each temperature, we also show the density plot of the local nematic order $\left\langle m_{\|, i}\right\rangle$ for a typical disorder realization. The density plots show well-defined domains of static positive (negative) nematic order perfectly coincident with the regions rich in horizontal (vertical) $J_{1}$-bond dilution. When the domains are bigger than $L, P\left(m_{\sigma}\right)$ exhibits two symmetric peaks, a feature observed in the clean (disorder-free) samples (not shown). Increasing $L$ for fixed impurity density and $T$, more and more domains fit inside the system and thus $P\left(m_{\sigma}\right)$ approaches a zeromean Gaussian of vanishing width $\sim 1 / L$ as dictates the central limit theorem.

\section{E. Disorder on the diagonal couplings}

For completeness, we have studied the effects of quenched disorder only on the next-nearest neighbor $J_{2}$ couplings. We have verified that the nematic-paramagnet transition is preserved. As discussed in Sec. IIID, this is expected since disorder on the $J_{2}$-couplings cannot generate random nematic fields. Based on universality, 


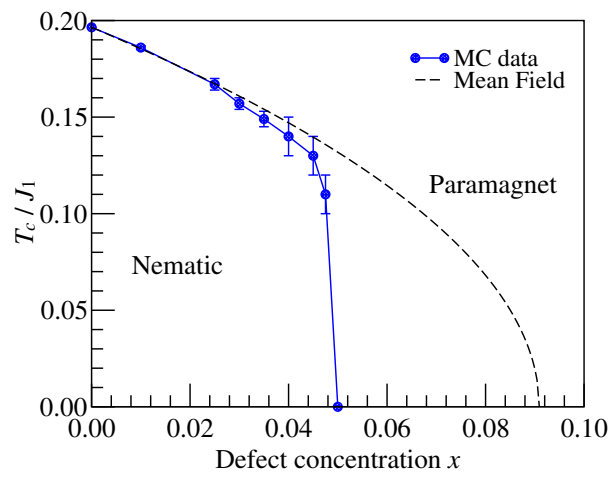

Figure 12. Critical temperature $T_{c}$ vs the dilution concentration of $J_{2}$ bonds $x$. The dashed line is the prediction given by mean field theory $T_{c}=0.62 \sqrt{\left(2(1-x) J_{2}-J_{1}\right) J_{1}}$. Here, $J_{2}=0.55 J_{1}$ and we have studied systems of sizes up to $L=80$. The MC critical temperature was estimated from the crossings of the Binder cumulant. The solid line is just a guide for the eyes.

we then expect this transition to belong to the usual Ising universality class furnished with logarithmic corrections [37-39]. Our results (not shown) are compatible with this scenario. ${ }^{11}$

In Fig. 12 we plot the critical temperature $T_{c}$ as a function of $x$, the density of diluted $J_{2}$ bonds. As before, we have set $J_{2}=0.55 J_{1}$. The critical temperature was obtained from the crossing of the nematic Binder cumulant (9). For lower densities $x \lesssim 2.5 \%$, the critical temperature follows a simple mean-field prediction as we explain below. At higher densities, the nematic order is considerably weakened and completely destroyed above $\approx 5 \%$.

For $0.5 J_{1} \leq J_{2} \lesssim 0.9 J_{1}$, the MC clean critical temperature obtained in Ref. 11 is well approximated by $T_{c}^{\text {(clean })} \approx A \sqrt{\left(2 J_{2}-J_{1}\right) J_{1}}$ with $A \approx 0.62$. In a simple mean-field approximation, we replace $J_{2}$ by its mean value $J_{2}(1-x)$. The mean-field critical temperature (shown as a dashed line in Fig. 12) is thus $T_{c} \approx A \sqrt{\left(2(1-x) J_{2}-J_{1}\right) J_{1}}$.

\section{F. The spin-vortex-crystal glass state and the spin-vorticity-density-wave order}

Finally, for completeness, we briefly study the disorder effects on the $T=0$ spin configuration obtained from the energy minimization method.

As discussed in Sec. III B, disorder perturbatively selects the SVC state. Non-perturbative effects, however, destroy this order (see Sec. IIIE). The vestigial SVDW order, on the order hand, is stable against weak disorder.

11 Evidently, we cannot exclude other scenarios such as nonuniversal disorder-dependent critical exponents since large system sizes (unreachable for our current numerical resources) are required [40].
Our aim is to confirm this prediction. Turns out that this is not a simple task because the amount of disorder required to destroy the coplanarity is not large and thus, large samples are required. We circumvent this obstacle by adding to the Hamiltonian (1) a positive biquadratic term $K \sum_{\langle i j\rangle}\left(\mathbf{S}_{i} \cdot \mathbf{S}_{j}\right)^{2}[10,20]$ with small $K=0.05$.

We have considered $J_{1}$-bond disorder such that $J_{1, i j}=$ $1+\Delta_{i j}$ with $\Delta_{i j}$ being either $\Delta$ or $-\Delta$ with equal probability. Thus, $\Delta$ parameterizes the disorder strength. The next-nearest neighbor interaction is kept fixed at $J_{2}=0.55$. In Fig. 13 we plot the SVDW axial order parameter (5) projected onto the $(z)$ direction perpendicular to the $(x y)$ coplanar spin configuration. Clearly, the SVDW order is finite for weak disorder $\Delta \leq \Delta_{c} \approx 0.2$. On the other hand, the SVDW order vanishes in the thermodynamic limit for $\Delta>\Delta_{c}$. We have verified (not shown) that the spin configuration is no longer coplanar in this regime. It is plausible that the destruction of the SVDW order coincides with the loss of coplanarity (and not before).

\section{CONCLUSIONS AND OUTLOOK}

In this section we summarize our results and discuss on their implications. Combining symmetry arguments, numerical minimization, and large-scale Monte Carlo simulations, we have revisited the square lattice $J_{1}-J_{2}$ classical Heisenberg model and have shown that long-range order at any finite temperature and in the frustrated orderby-disorder regime $2 J_{2}>J_{1}$ is perturbatively unstable against any finite density of generic bond and/or site disorder due to random-field effects. At zero temperature and low density of impurities, the state is a coplanar spin-vortex-crystal glass with finite spin-vorticitydensity-wave vestigial order.

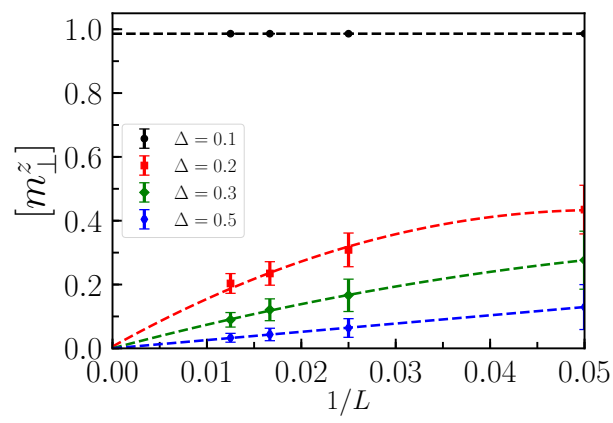

Figure 13. The $T=0$ disorder average of the $z$-component of the SVDW order parameter (5) as a function of the system size $L$ for various disorder strength $\Delta$ (see text). The spin configuration is on the $x y$ plane in the weak disorder limit $\Delta \leq \Delta \approx 0.2$ and the SVDW order is finite. For stronger disorder $\Delta>\Delta_{c}$, the spin configuration is no longer coplanar and $\left[m_{\perp}^{z}\right]$ vanishes as $L \rightarrow \infty$. 


\section{A. Equivalence between site and bond disorder}

It is well-known that a single bond defect and a site impurity have quite different effects as they select different states out of the ground-state manifold (stripe and spin-vortex crystal, respectively). We have shown that two nearest-neighbor site vacancies mimic a $J_{1}$-bond defect and, likewise, two site-sharing non-collinear $J_{1}$-bond defects mimic a site vacancy. Thus, we have established the equivalence between the effects of site and bond disorder when a finite density of impurities is considered. They both can select one of the stripe states or the SVC state, and induce random dipolar SVC fields and random nematic fields.

Interestingly, disorder on the $J_{2}$ bonds has a quite different effect. It does not lift the ground-state degeneracy and, thus, cannot induce any sort of symmetry-breaking random term. It can only induce random mass. Thus, long-range nematic order is stable against weak $J_{2}$-bond disorder.

We recall that in XY easy-plane pyrochlores, a related order-by-disorder frustrated system, the effects of site and bond disorder were numerically verified to be equivalent [13]. Finally, our equivalence extends the results of Ref. 12 (which states that generic non-collinear coplanar order is perturbatively unstable against bond disorder in $d \leq 2$ ) to site disorder as well.

\section{B. Random fields, random easy-axes and transverse dipolar fields}

In the ObD regime, both the perturbatively selected states (the collinear nematic at finite temperatures and the anti-collinear spin-vortex crystal at $T=0$ ) break a real-space symmetry. As a consequence, disorder generates random conjugate fields which have nonperturbative effects at $d \leq 2$. For the discrete symmetry stripe/nematic order, the generation of the random field is readily understood. Disorder locally breaks the same symmetry lifting the degeneracy between the equivalent ordered states. The order-parameter discrete symmetry character ensures that the generated field is short ranged, i.e., it is local. Notice that these arguments are not tied to the ObD mechanism. They apply to any phase where a real-space symmetry is spontaneously broken. An important example is the destruction of the stripe phase in the $J_{1}-J_{2}$ frustrated Ising magnets [8].

Fundamentally, the random easy axes discussed in the Appendix and the nematic random fields have the same common origin: impurities locally lifting the groundstate degeneracy. In the latter case, the disorder completely lifts the $\mathrm{Z}_{2}$ order-parameter degeneracy, and thus, can be recast as a random field. In the former, the order parameter $\mathrm{Z}_{3} \otimes \mathrm{Z}_{2}$ symmetry is broken down to $\mathrm{Z}_{2}$ and, thus, has the effects of random easy axes.

This symmetry-based strategy for determining the disorder effects in discrete-symmetry orders is very ap- pealing due to its simplicity. However, it is not totally clear whether it is relevant or useful to the case of inversion-symmetry-breaking order since this symmetry is not broken by a single $J_{1}$-bond defect (or a pair of nearest-neighbor vacancies). Instead, it locally releases frustration which acts as a local transverse field. Goldstone modes then communicate this perturbation as a slowly decaying dipolar field $\sim r^{1-d}$. Complicating even further this scenario, the effects on the associated spin-vorticity-density-wave vestigial order is much milder since the parent SVC "handness" is preserved (disorder acts as a weak SVDW $s$-wave field which decays $\left.\sim r^{-2 d}\right)$. While in $d \leq 2$ SVC order is completely destroyed, the SVDW order is perturbatively stable. Clearly, it is desirable to study the disorder effects on other non-collinear ground states. We have also verified that in the regime of strong disorder, the spin configuration becomes noncoplanar. Describing and understanding how disorder destroys coplanarity is a task left for future research.

Recently, it was noted that local correlations on the disorder variables can prevent random mass and, consequently, Griffiths singularities on a phase transition [41]. More recently [42], it was shown that local correlations on the disordered variables can also prevent random stripe fields as well. This is also possible in the model Hamiltonian (1) if, for instance, two vacancies are forbidden to be nearest neighbors. Thus, there will be no generation of local nematic fields and of transverse dipolar fields. In sum, this class of local correlation in the disorder variables can prevent the generation of random fields ensuring the phase transition and the low-temperature long-range nematic order.

In experiments, bond defects induced by chemical doping distorting the local lattice may induce similar vertical and horizontal local bond defects. This can either prevent or greatly diminish the amplitude of the random fields and, therefore, the phase transition may either be preserved or appear to be preserved. ${ }^{12}$ Finally, we mention the recently synthesized $\mathrm{Sr}_{2} \mathrm{CuTe}_{1-x} \mathrm{~W}_{x} \mathrm{O}_{6}$ compound which is a quasi-two-dimensional spin- $1 / 2$ magnet modeled by the Hamiltonian (1) [44]. Disorder in this compound is of random-couplings type [45]. Interestingly, the couplings appear equally disordered in the plaquette. In other words, it realizes the correlated disorder mechanism above mentioned. In agreement with our results, the nematic phase is quite robust against weak disorder. It is worthy noting that our results also explains the numerical findings of Ref. 46.

\section{Quantum mechanical effects}

We have established that the classical (finite- $T$ ) paramagnet-nematic phase transition is precluded by

12 An analogous result was obtained for the case of random mass [43]. 
generic quenched disorder (i.e., disorder which includes random $J_{1}$ bonds). Since quantum fluctuations are expected to play no role on the critical behavior of this finite- $T$ transition, it is very plausible that our result directly applies to quantum mechanical systems as well.

We have also shown that the resulting paramagnet is polarized in the glassy nematic order for all temperatures, i.e., the system is broken into domains of local nematic order. How is this paramagnet changed by quantum fluctuations? Deep in the frustrated regime $J_{2} \gg J_{1}$ where the semi-classical approach is expected to be valid, the naive expectation is that the local nematic domains are not melted by quantum fluctuations since they also select the stripe state via the order-by-(quantum)disorder mechanism [10]. ${ }^{13}$ We also expect a weaker SVC and SVDW response at lower temperatures because the size of the nematic domains will not vanish as $T \rightarrow 0$.

Our results are thus relevant for a wide range of materials such as ( $\mathrm{K}$ or $\mathrm{Rb}) \mathrm{MoOPO}_{4} \mathrm{Cl}$ [47], $\mathrm{Li}_{2} \mathrm{VO}(\mathrm{Si}$ or $\mathrm{Ge}) \mathrm{O}_{4}[48,49]$, and $\operatorname{abVO}\left(\mathrm{PO}_{4}\right)_{2}$ (with ab $=\mathrm{BaCd}$, $\mathrm{SrZn}, \mathrm{BaZn}, \mathrm{Pb}_{2}, \mathrm{PbZn}$ ) [50-53]. They are modeled by the Hamiltonian (1) and disorder effects can be introduced by chemical substitution.

Around the antiferromagnetic-stripe quantum phase transition $0.4 \lesssim J_{2} / J_{1} \lesssim 0.6$, the system does not display long-range order and its description lies beyond the reach of the semi-classical approach [54-57]. The effects of disorder on the phases appearing in that interval is an important question which has received considerable attention recently [58-63].

\section{ACKNOWLEDGMENTS}

We thank Pedro Consoli, Santanu Dey, Matthias Vojta, André Vieira, Eduardo Miranda and Rafael Fernandes for useful discussions and collaborations on related topics. We also thank N. P. Teodosio for collaboration at an early stage of this project. This work was supported by the CAPES - Finance Code 001, by FAPESP Grants No. 2015/23849-7, No. 2016/108261, No. 2019/17026-9, and No. 2019/17645-0, by CNPq Grants No. 307548/2015-5, No. 406399/2018-2, and No. 302994/2019-0, by the Deutsche Forschungsgemeinschaft (DFG) through SFB 1143 (project id 247310070), the Würzburg-Dresden Cluster of Excellence ct.qmat (EXC 2147, Project ID No. 390858490), and by the IMPRS for Chemistry and Physics of Quantum Materials at MPICPfS.

13 This is certainly valid even beyond the semi-classical approximation in a temperature window ranging from higher temperatures down to slightly below the clean critical temperature.

\section{Appendix A: Random easy-axes}

Several recent works $[12,13,42,64,65]$ have discussed specific examples of frustrated magnetic systems in which the effects of interaction-symmetry-preserving disorder can be understood in terms of effective randomfields $[8,9,66]$. Here, we show that effective random axes $[33,67]$ are generated by site and bond disorder in easy-plane pyrochlores providing a clear physical interpretation of the numerical data. The important issue is that these new terms have much more dramatic effects such as precluding any phase transition at $d \leq 2$, or even stabilizing a cluster-spin glass phase at $d \geq 3$.

Our strategy is straightforward. As discussed in Secs. (III A) and (IIIE) we simply analyze how a single impurity lifts the ground-state degeneracy.

The easy-plane pyrochlores are XY frustrated magnets interacting via diagonal and off-diagonal (spin-orbit induced) interactions which can be modeled by the system Hamiltonian [68]

$\mathcal{H}=-\sum_{\langle j k\rangle}\left[J_{j k}^{x x} S_{j}^{x} S_{k}^{x}+J_{j k}^{y y} S_{j}^{y} S_{k}^{y}+J_{j k}^{x y}\left(S_{j}^{x} S_{k}^{y}+S_{j}^{y} S_{k}^{x}\right)\right]$,

where the sum runs over pairs of nearest-neighbor sites on a cubic pyrochlore lattice (see Fig. 14), the coupling constants are

$$
J_{j k}^{x x(y y)}=J_{j k}\left(1 \mp \alpha_{j k} \cos \gamma_{j k}\right), J_{j k}^{x y}=J_{j k} \alpha_{j k} \sin \gamma_{j k},
$$

the classical spins $\mathbf{S}_{j}=\left(\cos \theta_{j}, \sin \theta_{j}\right)$ are 2-component unity vectors, $J_{j k}$ parameterizes the local energy scale, and $\alpha_{j k}$ parameterizes the relative strength of the local spin-orbit coupling. The direction-dependent angles are $\gamma_{01}=\gamma_{23}=\gamma_{04}=\gamma_{56}=0, \gamma_{02}=\gamma_{13}=\gamma_{05}=\gamma_{46}=$ $2 \pi / 3$, and $\gamma_{03}=\gamma_{12}=\gamma_{06}=\gamma_{45}=-2 \pi / 3$.

In the clean limit $\left(J_{j k}=J\right.$ and $\left.\alpha_{j k}=\alpha\right)$ and for $-2<\alpha<2$, the ground state manifold is $\mathrm{O}(2)$ acciden-

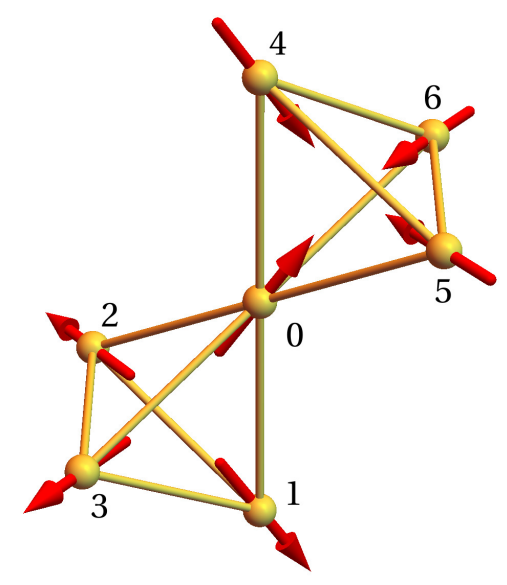

Figure 14. The sketch of the six nearest-neighbors sites in a pyrochlore lattice. The local $z$ axes are also shown as red arrows. For clarity, the local $x$ and $y$ axes are not shown. 
tally degenerate being a ferromagnetic state (in the local reference frame) with spins pointing along any direction $\theta_{j}=\theta$ in the XY plane. The ground-state energy $E_{0}=$ $-3 J N$ is $\alpha$ independent. As can be expected, the orderby-(thermal/quantum-)disorder mechanism lifts the $\mathrm{O}(2)$ ground-state degeneracy down to $\mathrm{Z}_{6}$ : for $0<\alpha<2$ $(-2<\alpha<0)$ the dubbed $\psi_{2}\left(\psi_{3}\right)$ state is selected which corresponds to spins pointing along one of the $\cos \left(\frac{\pi}{3} n\right) \hat{x}+\sin \left(\frac{\pi}{3} n\right) \hat{y}\left[\cos \left(\frac{\pi}{3} n+\frac{\pi}{6}\right) \hat{x}+\sin \left(\frac{\pi}{3} n+\frac{\pi}{6}\right) \hat{y}\right]$ directions, with $n=0, \ldots, 5$, in the local reference frame $[69,70]$.

The bare interaction symmetry is actually a simple $\mathrm{Z}_{2}$ one $\left(\theta_{j} \rightarrow \theta_{j}+\pi\right)$. This becomes evident when rewriting the local interaction energy as

$$
\mathcal{H}_{j k}=-J_{j k}\left(\cos \left(\theta_{j}-\theta_{k}\right)-\alpha_{j k} \cos \left(\theta_{j}+\theta_{k}+\gamma_{j k}\right)\right) .
$$

The symmetry is enhanced to $\mathrm{Z}_{6}$ when there is a realspace equivalence between the three axes connecting the site pairs $(0,1),(0,2)$, and $(0,3)$, i.e., $\left(\theta_{j} \rightarrow \theta_{j}+\frac{\pi}{3}\right.$ and $\left.\mathcal{H}_{01} \rightarrow \mathcal{H}_{02} \rightarrow \mathcal{H}_{03} \rightarrow \mathcal{H}_{01}\right)$. Not totally surprisingly, thermal and/or quantum fluctuations lift the $\mathrm{O}(2)$ ground-state degeneracy down to $\mathrm{Z}_{6}$ in the clean system. Likewise, quenched disorder lifts down to a $\mathrm{Z}_{2}$ manifold as shown explicitly in the following. Consider a single bond defect between sites $j$ and $k$ such that $J_{j k}=J+\delta J_{j k}$ and, for simplicity, $\alpha_{j k}=\alpha$. Thus, the ground-state degenerate energy $E_{0}$ is changed by

$$
\delta E=\delta J_{j k}\left(\alpha \cos \left(2 \theta+\gamma_{j k}\right)-1\right)
$$

Hence, a single bond defect locally lifts the accidental $\mathrm{O}(2)$ ground-state manifold down to a $\mathrm{Z}_{2}$ manifold, the same one of the interactions. Consequently, the generated term in the field theory is a random axis. For $\delta J_{j k} \alpha$ negative (positive), the preferred axis is in the direction $-\frac{\gamma_{j k}}{2}\left(\frac{\pi-\gamma_{j k}}{2}\right)$ which is one of the three axis of the $\psi_{2}$ $\left(\psi_{3}\right)$ set of states.

A single-site defect, on the other hand, does not lift the degeneracy between the three different axes and, therefore, does not generate any easy-axis term. Two nearestneighbor site defects, on the other hand, do break this symmetry generating the random easy-axis term. Thus, the equivalence between the effects of site and bond disorder is restored when a finite density of impurities is considered.

What is the resulting state? In the absence of disorder and in the ObD regime, the $\psi_{2,3}$ fluctuations at low temperatures can be modeled by a short-ranged $\mathrm{Z}_{6}$ planar clock model. In the presence of weak disorder, this model must be furnished with local random axes. The Imry-Ma criterion [5] directly applies for this case of random axes. Thus, the lower critical dimension is $d_{c}^{-}=2$. Since $d=3$, then long-range $\psi_{2,3}$ order is stable against weak disorder. For strong disorder, the system thus breaks into local domains. Very likely, the effective $\alpha$ throughout the system has zero-mean (or larger variance compared to its mean value), and thus, the domains of $\psi_{2}$ and $\psi_{3}$ types are equally (or almost equally) probable. In other words, there are six equally probable random easy axes.

There is an important difference with respect to the nematic case discussed in Sec. IIID. Here, the domains have two equally probable states favored by the local easy axis. Thus, at sufficiently high temperatures, the local $\psi_{2(3)}$ order is vanishing. In addition, there are an exponentially large number of equivalent ground states. ${ }^{14}$ For these reasons, it is plausible to expect a transition to a spin-cluster glass phase at low temperatures. All these conclusions were confirmed numerically in Refs. 13, 71.

Finally, notice that the same disorder-induced randomaxes term appears in the two-dimensional analog of the model (A1) on the triangular lattice with the three different $\gamma_{i j}$-angles being related to the interactions along the $0, \pm \frac{2 \pi}{3}$ directions. In $d=d_{c}^{-}=2$, however, longrange order is precluded by any amount of disorder. For zero-mean disorder $\left[\delta J_{j k}\right]=0$, stripe domains on the six directions $n \frac{\pi}{3}(n=0,1, \ldots, 5)$ are expected. ${ }^{15} \mathrm{~A}$ glassy state, however, may be melted by thermal fluctuations as the lower critical dimension of the problem may be 5/2 [72]. Quantum fluctuations, on the other hand, may not be sufficiently strong to destroy the glassy order (which is compatible with the results of Ref. 64). The understanding of the disorder effects as inducing the formation of domains of different stripe orders were previously reported in Ref. 65.

Although we have focused on easy-plane pyrochlores, our arguments also apply other similar models interacting via strong anisotropic interactions such as HeisenbergKitaev model $[73,74]$. In those cases, the ground state exhibits $\mathrm{Z}_{6}$ degeneracy which is locally lifted by disorder.
${ }^{14}$ Evidently, there is an exponentially weak effective coupling between these domains which are unimportant at finite temperatures.

15 We find interesting to imagine that, if there was order-bydisorder in this system selecting, say, the even- $n$ domains, then, the odd- $n$ domains would be penalized. The symmetry would be restore only at stronger disorder.
[1] Robert B. Griffiths, "Nonanalytic behavior above the critical point in a random ising ferromagnet," Phys. Rev. Lett. 23, 17-19 (1969).

[2] Barry M. McCoy, "Incompleteness of the critical exponent description for ferromagnetic systems containing random impurities," Phys. Rev. Lett. 23, 383-386 (1969).

[3] A. B. Harris, "Effect of random defects on the critical behaviour of ising models," J. Phys. C 7, 1671 (1974). 
[4] Yoseph Imry and Michael Wortis, "Influence of quenched impurities on first-order phase transitions," Phys. Rev. B 19, 3580-3585 (1979).

[5] Y. Imry and S-k Ma, "Random-Field Instability of the Ordered State of Continuous Symmetry," Phys. Rev. Lett. 35, 1399 (1975).

[6] Thomas Vojta, "Disorder in quantum many-body systems," Annu. Rev. Cond. Matt. Phys. 10, 233-252 (2019).

[7] T. Vojta and J. A. Hoyos, "Criticality and Quenched Disorder: Harris Criterion Versus Rare Regions," Phys. Rev. Lett. 112, 075702 (2014).

[8] J. F Fernandez, "Random fields generated by dilution in zero external field," Europhysics Letters (EPL) 5, 129133 (1988).

[9] Y V Fyodorov and E F Shender, "Random-field effects in antiferromagnets with classically degenerate ground states," Journal of Physics: Condensed Matter 3, 91239128 (1991).

[10] C. L. Henley, "Ordering due to disorder in a frustrated vector antiferromagnet," Phys. Rev. Lett. 62, 2056 (1989).

[11] C. Weber and F. Mila, "Anticollinear magnetic order induced by impurities in the frustrated Heisenberg model of pnictides," Phys. Rev. B 86, 184432 (2012).

[12] S. Dey, E. C. Andrade, and M. Vojta, "Destruction of long-range order in noncollinear two-dimensional antiferromagnets by random-bond disorder," Phys. Rev. B 101, 020411(R) (2020).

[13] E. C. Andrade, J. A. Hoyos, S. Rachel, and M. Vojta, "Cluster-Glass Phase in Pyrochlore $X Y$ Antiferromagnets with Quenched Disorder," Phys. Rev. Lett. 120, 097204 (2018).

[14] P. Chandra, P. Coleman, and A. I. Larkin, "Ising transition in frustrated heisenberg models," Phys. Rev. Lett. 64, 88 (1990).

[15] C. Weber, L. Capriotti, G. Misguich, F. Becca, M. Elhajal, and F. Mila, "Ising Transition Driven by Frustration in a 2D Classical Model with Continuous Symmetry," Phys. Rev. Lett. 91, 177202 (2003).

[16] C. Lacroix, P. Mendels, and F. Mila, eds., Introduction to Frustrated Magnetism (Springer, Berlin, 2011).

[17] J. Villain, R. Bidaux, J.-P. Carton, and R. Conte, "Order as an effect of disorder," J. Phys. (Paris) 41, 1263 (1980).

[18] E.F. Shender, "Antiferromagnetic garnets with fluctuationally interacting sublattices," Sov. Phys. JETP 56, 178 (1982).

[19] N. D. Mermin and H. Wagner, "Absence of ferromagnetism or antiferromagnetism in one- or two-dimensional isotropic heisenberg models," Phys. Rev. Lett. 17, 11331136 (1966).

[20] R. M. Fernandes, S. A. Kivelson, and E. Berg, "Vestigial chiral and charge orders from bidirectional spin-density waves: Application to the iron-based superconductors," Phys. Rev. B 93, 014511 (2016).

[21] L. R. Walker and R. E. Walstedt, "Computer model of metallic spin-glasses," Phys. Rev. Lett. 38, 514 (1977).

[22] A. Andreanov and P. A. McClarty, "Order induced by dilution in pyrochlore XY antiferromagnets," Phys. Rev. B 91, 064401 (2015).

[23] V. S. Maryasin and M. E. Zhitomirsky, "Order from structural disorder in the $X Y$ pyrochlore antiferromagnet $\mathrm{Er}_{2} \mathrm{Ti}_{2} \mathrm{O}_{7}, "$ Phys. Rev. B 90, 094412 (2014).

[24] R. M. Fernandes, P. P. Orth, and J. Schmalian, "Inter- twined vestigial order in quantum materials: Nematicity and beyond," Annu. Rev. Cond. Matt. Phys. 10, 133 (2019).

[25] G. Grinstein and Shang-keng Ma, "Roughening and lower critical dimension in the random-field ising model," Phys. Rev. Lett. 49, 685-688 (1982).

[26] G. Grinstein and Shang-keng Ma, "Surface tension, roughening, and lower critical dimension in the randomfield ising model," Phys. Rev. B 28, 2588-2601 (1983).

[27] J. Villain, "Commensurate-incommensurate transition with frozen impurities," Journal de Physique Lettres 43, 551-558 (1982).

[28] J. Villain, "Insulating spin glasses," Z. Phys. B 33, 31 (1979).

[29] Florent Krzakala, Federico Ricci-Tersenghi, and Lenka Zdeborová, "Elusive spin-glass phase in the random field ising model," Phys. Rev. Lett. 104, 207208 (2010).

[30] Y Miyatake, M Yamamoto, J J Kim, M Toyonaga, and O Nagai, "On the implementation of theheat bath algorithms for monte carlo simulations of classical heisenberg spin systems," Journal of Physics C: Solid State Physics 19, 2539-2546 (1986).

[31] Michael Creutz, "Overrelaxation and monte carlo simulation," Phys. Rev. D 36, 515-519 (1987).

[32] Koji Hukushima and Koji Nemoto, "Exchange monte carlo method and application to spin glass simulations," Journal of the Physical Society of Japan 65, 1604-1608 (1996).

[33] K. H. Fischer and J. A. Hertz, Spin Glasses (Cambridge University Press, Cambridge, 1991).

[34] J. H. Pixley and A. P. Young, "Large-scale Monte Carlo simulations of the three-dimensional $X Y$ spin glass," Phys. Rev. B 78, 014419 (2008).

[35] Nigel Gondenfeld, Lectures on phase transitions and renormalization group, Frontiers in Physics v. 85 (Perseus Books Publishing, Reading, EUA, 1992) p. 394.

[36] Anders W. Sandvik, "Computational studies of quantum spin systems," AIP Conference Proceedings 1297, 135338 (2010).

[37] Viktor S. Dotsenko and Vladimir S. Dotsenko, "Critical behaviour of the phase transition in the $2 \mathrm{~d}$ ising model with impurities," Advances in Physics 32, 129172 (1983).

[38] B. N. Shalaev, "Critical behavior of the two-dimensional ising model with random bonds," Physics Reports 237, 129-188 (1994).

[39] R. Shankar, "Exact critical behavior of a random bond two-dimensional ising model," Phys. Rev. Lett. 58, 24662469 (1987).

[40] Q. Zhu, X. Wan, R. Narayanan, J. A. Hoyos, and T. Vojta, "Emerging criticality in the disordered three-color ashkin-teller model," Phys. Rev. B 91, 224201 (2015).

[41] José A. Hoyos, N. Laflorencie, A. P. Vieira, and T. Vojta, "Protecting clean critical points by local disorder correlations," Europhys. Lett. 93, 30004 (2011).

[42] S. Singh Kunwar, A. Sen, T. Vojta, and R. Narayanan, "Tuning a random-field mechanism in a frustrated magnet," Phys. Rev. B 98, 024206 (2018).

[43] J. C. Getelina, F. C. Alcaraz, and J. A. Hoyos, "Entanglement properties of correlated random spin chains and similarities with conformally invariant systems," Phys. Rev. B 93, 045136 (2016).

[44] O. Mustonen, S. Vasala, K. P. Schmidt, E. Sadrollahi, H. C. Walker, I. Terasaki, F. J. Litterst, E. Baggio- 
Saitovitch, and M. Karppinen, "Tuning the $s=1 / 2$ square-lattice antiferromagnet $\mathrm{Sr}_{2} \mathrm{Cu}\left(\mathrm{Te}_{1-x} \mathrm{w}_{x}\right) \mathrm{O}_{6}$ from néel order to quantum disorder to columnar order," Phys. Rev. B 98, 064411 (2018).

[45] Vamshi M. Katukuri, P. Babkevich, O. Mustonen, H. C. Walker, B. Fåk, S. Vasala, M. Karppinen, H. M. Rønnow, and O. V. Yazyev, "Exchange interactions mediated by nonmagnetic cations in double perovskites," Phys. Rev. Lett. 124, 077202 (2020).

[46] Wenshan Hong, Lu Liu, Chang Liu, Xiaoyan Ma, Akihiro Koda, Xin Li, Jianming Song, Wenyun Yang, Jinbo Yang, Peng Cheng, Hongxia Zhang, Wei Bao, Xiaobai Ma, Dongfeng Chen, Kai Sun, Wenan Guo, Huiqian Luo, Anders W. Sandvik, and Shiliang Li, "Extreme suppression of antiferromagnetic order and critical scaling in a two-dimensional random quantum magnet," Phys. Rev. Lett. 126, 037201 (2021).

[47] Hajime Ishikawa, Nanako Nakamura, Makoto Yoshida, Masashi Takigawa, Peter Babkevich, Navid Qureshi, Henrik M. Rønnow, Takeshi Yajima, and Zenji Hiroi, " $J_{1}-J_{2}$ square-lattice Heisenberg antiferromagnets with $4 d^{1}$ spins: $A \mathrm{MoOPO}_{4} \mathrm{Cl}(A=\mathrm{K}, \mathrm{Rb})$," Phys. Rev. B 95, 064408 (2017).

[48] R. Melzi, S. Aldrovandi, F. Tedoldi, P. Carretta, P. Millet, and F. Mila, "Magnetic and thermodynamic properties of $\mathrm{Li}_{2} \mathrm{VOSiO}_{4}$ : A two-dimensional $S=1 / 2$ frustrated antiferromagnet on a square lattice," Phys. Rev. B 64, 024409 (2001).

[49] H. Rosner, R. R. P. Singh, W. H. Zheng, J. Oitmaa, and W. E. Pickett, "High-temperature expansions for the $J_{1}-J_{2}$ Heisenberg models: Applications to ab initio calculated models for $\mathrm{Li}_{2} \mathrm{VOSiO}_{4}$ and $\mathrm{Li}_{2} \mathrm{VOGeO}_{4}$," Phys. Rev. B 67, 014416 (2003).

[50] R. Nath, A. A. Tsirlin, H. Rosner, and C. Geibel, "Magnetic properties of $\mathrm{BaCdVO}\left(\mathrm{PO}_{4}\right)_{2}$ : A strongly frustrated spin- $\frac{1}{2}$ square lattice close to the quantum critical regime," Phys. Rev. B 78, 064422 (2008).

[51] E.E. Kaul, H. Rosner, N. Shannon, R.V. Shpanchenko, and C. Geibel, "Evidence for a frustrated square lattice with ferromagnetic nearest-neighbor interaction in the new compound $\mathrm{Pb}_{2} \mathrm{VO}\left(\mathrm{PO}_{4}\right)_{2}$," Journal of Magnetism and Magnetic Materials 272-276, 922-923 (2004), proceedings of the International Conference on Magnetism (ICM 2003).

[52] Alexander A. Tsirlin and Helge Rosner, "Extension of the spin- $\frac{1}{2}$ frustrated square lattice model: The case of layered vanadium phosphates," Phys. Rev. B 79, 214417 (2009).

[53] Alexander A. Tsirlin, Ramesh Nath, Artem M. Abakumov, Roman V. Shpanchenko, Christoph Geibel, and Helge Rosner, "Frustrated square lattice with spatial anisotropy: Crystal structure and magnetic properties of PbZnVO(PO $\left(\mathrm{PO}_{4}\right)_{2}$," Phys. Rev. B 81, 174424 (2010).

[54] H.-C. Jiang, H. Yao, and L. Balents, "Spin liquid ground state of the spin- $\frac{1}{2}$ square $J_{1}-J_{2}$ Heisenberg model," Phys. Rev. B 86, 024424 (2012).

[55] S.-S. Gong, W. Zhu, D. N. Sheng, O. I. Motrunich, and M. P. A. Fisher, "Plaquette Ordered Phase and Quantum Phase Diagram in the Spin- $\frac{1}{2} J_{1}-J_{2}$ Square Heisenberg Model," Phys. Rev. Lett. 113, 027201 (2014).

[56] Satoshi Morita, Ryui Kaneko, and Masatoshi Imada, "Quantum spin liquid in spin-1/2 j1-j2 heisenberg model on square lattice: Many-variable variational monte carlo study combined with quantum-number projections,"
Journal of the Physical Society of Japan 84, 024720 (2015).

[57] L. Wang and A. W. Sandvik, "Critical Level Crossings and Gapless Spin Liquid in the Square-Lattice Spin-1/2 $J_{1}-J_{2}$ Heisenberg Antiferromagnet," Phys. Rev. Lett. 121, 107202 (2018).

[58] K. Uematsu and H. Kawamura, "Randomness-induced quantum spin liquid behavior in the $s=\frac{1}{2}$ random $J_{1}-J_{2}$ Heisenberg antiferromagnet on the square lattice," Phys. Rev. B 98, 134427 (2018).

[59] H. Kawamura and K. Uematsu, "Nature of the randomness-induced quantum spin liquids in two dimensions," J. Phys.: Condens. Matter 31, 504003 (2019).

[60] L. Liu, H. Shao, Y.-C. Lin, W. Guo, and A. W. Sandvik, "Random-singlet phase in disordered two-dimensional quantum magnets," Phys. Rev. X 8, 041040 (2018).

[61] I. Kimchi, A. Nahum, and T. Senthil, "Valence bonds in random quantum magnets: Theory and application to ybmggao 4 ," Phys. Rev. X 8, 031028 (2018).

[62] Seung-Ho Baek, Hyeon Woo Yeo, Seung-Hwan Do, Kwang-Yong Choi, Lukas Janssen, Matthias Vojta, and Bernd Büchner, "Observation of a random singlet state in a diluted Kitaev honeycomb material," Phys. Rev. B 102, 094407 (2020).

[63] Lu Liu, Wenan Guo, and Anders W. Sandvik, "Quantum-critical scaling properties of the twodimensional random-singlet state," Phys. Rev. B 102, 054443 (2020).

[64] Z. Zhu, P. A. Maksimov, S. R. White, and A. L. Chernyshev, "Disorder-Induced Mimicry of a Spin Liquid in $\mathrm{YbMgGaO}_{4}$," Phys. Rev. Lett. 119, 157201 (2017).

[65] E. Parker and L. Balents, "Finite-temperature behavior of a classical spin-orbit-coupled model for $\mathrm{YbMgGaO}_{4}$ with and without bond disorder," Phys. Rev. B 97, 184413 (2018).

[66] A. Aharony, "Absence of Ferromagnetic Long Range Order in Random Isotropic Dipolar Magnets and in similar systems," Solid State Commun. 28, 667 (1978).

[67] Amnon Aharony, "Critical phenomena in systems with random anisotropies," Journal of Magnetism and Magnetic Materials 31-34, 1432-1434 (1983).

[68] K. A. Ross, L. Savary, B. D. Gaulin, and L. Balents, "Quantum Excitations in Quantum Spin Ice," Phys. Rev. X 1, 021002 (2011).

[69] M. E. Zhitomirsky, M. V. Gvozdikova, P. C. W. Holdsworth, and R. Moessner, "Quantum Order by Disorder and Accidental Soft Mode in $\mathrm{Er}_{2} \mathrm{Ti}_{2} \mathrm{O}_{7}$, , Phys. Rev. Lett. 109, 077204 (2012).

[70] L. Savary, K. A. Ross, B. D. Gaulin, J. P. C. Ruff, and L. Balents, "Order by Quantum Disorder in $\mathrm{Er}_{2} \mathrm{Ti}_{2} \mathrm{O}_{7}$," Phys. Rev. Lett. 109, 167201 (2012).

[71] R. Sarkar, J. W. Krizan, F. Brückner, E. C. Andrade, S. Rachel, M. Vojta, R. J. Cava, and H.-H. Klauss, "Spin freezing in the disordered pyrochlore magnet $\mathrm{NaCaCo}_{2} \mathrm{~F}_{7}$ : NMR studies and Monte Carlo simulations," Phys. Rev. B 96, 235117 (2017).

[72] Andrea Maiorano and Giorgio Parisi, "Support for the value $5 / 2$ for the spin glass lower critical dimension at zero magnetic field," Proceedings of the National Academy of Sciences 115, 5129-5134 (2018).

[73] Craig C. Price and Natalia B. Perkins, "Critical properties of the kitaev-heisenberg model," Phys. Rev. Lett. 109, 187201 (2012).

[74] E. C. Andrade and M. Vojta, "Magnetism in spin models 
for depleted honeycomb-lattice iridates: Spin-glass order towards percolation," Phys. Rev. B 90, 205112 (2014). 